\title{
Klodian Coko
}

\section{Jean Perrin and the Philosophers' Stories: The Role of Multiple Determination in Determining Avogadro's Number}

\begin{abstract}
The French physicist Jean Baptiste Perrin is widely credited with providing the conclusive argument for atomism. The most well-known part of Perrin's argument is his description of thirteen different procedures for determining Avogadro's number $(N)$-the number of atoms, ions, and molecules contained in a gram-atom, gram-ion, and gram-mole of a substance, respectively. Because of its success in ending the atomism debates, Perrin's argument has been the focus of much philosophical interest. The various philosophers, however, have reached different conclusions, not only about the argument's general rationale but also the role that the multiple determination of $N$ played in it. This paper emphasizes the historical development of Perrin's experimental work in order to understand the role that the multiple determination of molecular magnitudes played in his argument for molecular reality. It claims that Perrin used the multiple determination strategy to put forward an exceptionally strong no-coincidence argument to argue for both the correctness of the values for the molecular magnitudes determined and the validity of the auxiliary assumptions upon which the different determinations were based. The historicist approach also allows the identification of the elements responsible for the epistemic strength of Perrin's no-coincidence argument.
\end{abstract}




\section{Introduction}

The French physicist Jean Baptiste Perrin (1870-1942) is widely credited with providing the conclusive argument for atomism (Perrin 1913b; Brush 1968; Nye 1972; Chalmers 2009). The most well-known part of Perrin's argument is his description of thirteen different procedures for determining Avogadro's number $(N)$-the number of molecules, atoms, and ions contained in a gram-mole, gram-atom, and gram-ion of a substance, respectively, under the same conditions of pressure and temperature. The different procedures included Perrin's own three, which were based on the experimental study of the vertical distribution, mean displacement, and mean rotation of Brownian particles (Perrin 1909c, 1913b). The chart in Fig.1 appeared in the concluding section of Perrin's influential monograph Mouvement Brownien et Réalité Moléculaire (Perrin 1909c). In this monograph Perrin presented for the first time to a wide readership his experimental work on Brownian movement and its relationship with the existing evidence for the atomic-molecular conception of matter.

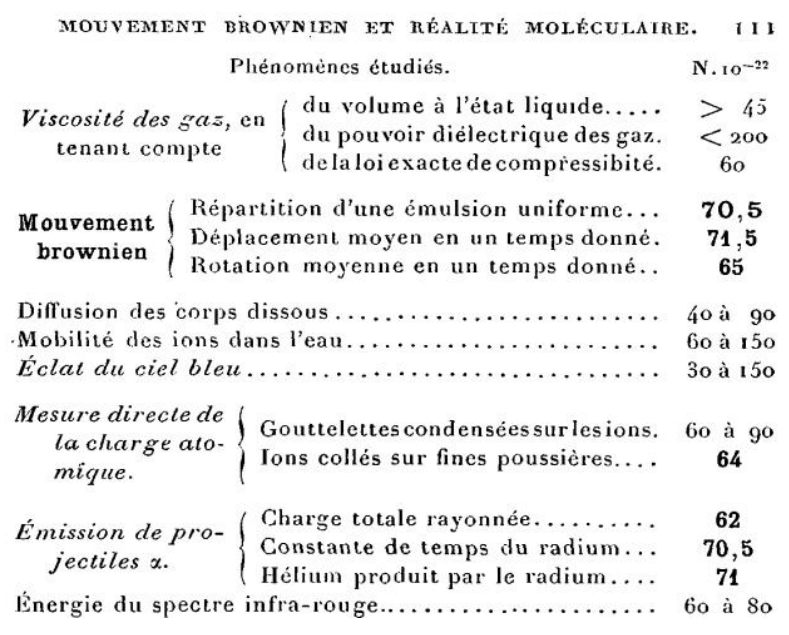

Fig.1

The chart summarizes the values for $N$ determined from the independent consideration of different phenomena such as viscosity of gases, Brownian movement, diffusion of dissolved 
bodies, alpha particle decay, charge of the electron, etc. ${ }^{1}$ Despite the slight variations and approximations, the different determinations seemed to agree with one another and, according to Perrin, suggested as the most probable value for $N$ the number $70,5.10^{22}$-which was also the value obtained in his own experimental study of the vertical distribution of Brownian particles. Perrin argued that this numerical agreement left no reasonable doubt about the validity of the atomic-molecular hypothesis. ${ }^{2}$ The agreement on the values for molecular magnitudes obtained by independent determinations was crucial for convincing (almost) all members of the scientific community for the reality of atoms and molecules, by 1913. For his experimental work in proving the discontinuous structure of matter Perrin was awarded the Nobel Prize in Physics, in 1926 (Perrin 1926).

Because it was very successful in ending nearly one hundred years of scientific debates over the existence of unobservable atoms and molecules, Perrin's argument has been the focus of much philosophical interest. We can discern two, relatively independent, philosophical treatments. On the one hand, Perrin's argument is often cited in philosophical discussions over the epistemic strategy of multiple determination-i.e., the epistemic strategy of using independent procedures to establish the same result. In this context, Perrin's argument is presented as a nocoincidence argument and its blunt rationale as being something along these lines: it would be a highly improbable coincidence for thirteen different procedures to converge at the same value for the number of molecules contained in a unit of substance and yet for molecules not to exist. On the other hand, there are philosophers who have looked more closely at Perrin's argument. These philosophers, however, have arrived at different conclusions, not only about the argument's general rationale but also the role that the multiple determination of $N$ played in it. 
In this paper I investigate the role that the multiple determination of molecular magnitudes played in Perrin's experimental work on Brownian movement and in his argument for molecular reality. I examine the traditional philosophical approaches to Perrin's case, and I indicate their shortcomings. In my account, I emphasize both the historical context and the historical development of Perrin's argument. Following this (historicist) approach, I locate the main elements responsible for the argument's epistemic strength. I argue that Perrin's argument was the result of his clear understanding of the philosophical and scientific challenges facing the empirical verification of claims regarding the existence of unobservable entities-such as atoms and molecules-at the beginning of the twentieth century. Perrin's efforts were influenced by the late nineteenth century recognition that an experiment in physics, in general, and the experimental investigation of unobservable entities, in particular, required the use of complex instruments and experimental procedures as well as the employment of many (theoretical and experimental) auxiliary assumptions. In lack of direct observable evidence for the existence of atoms and molecules, Perrin efforts were directed towards what he considered to be the next best thing: the determination of the various (hypothetical) molecular magnitudes via independently theory-dependent routes. The extremely remarkable agreement on the values for molecular magnitudes determined by independently theory-dependent procedures gave rise to an exceptionally strong no-coincidence argument that was used to argue for both the correctness of the determined values and the validity of the auxiliary assumptions upon which the different determinations were based. The structure of this no-coincidence argument, however, was more complex than the one encountered in the blunt rationale for multiple determination. There are structural elements in Perrin's argument which, although neglected in the various philosophical accounts, were responsible for its strength and, ultimately, its success. They are: (a) the 
numerical (or quantitative) nature of the agreement, (b) the very close agreement achieved (especially if one took into account the a priori improbability of such a close agreement), (c) the theoretical independence of the determination procedures, (d) the genetic (or historical) independence of both the determination procedures and of the agreement achieved, (e) the variety of the phenomena and molecular properties upon which the various determinations were based, (f) the high quality and reliability of some of the determinations, (g) the (final) lack of discordant results, (h) the ability to conclusively explain away objections and discordant results when they eventually emerged, and (i) the large number of concordant determinations.

The paper is structured as follows. In Section 2, I examine the traditional philosophical approaches to Perrin's argument, and I indicate their shortcomings. In Section 3, I provide a brief summary of the nineteenth century atomism debates and the scientific and philosophical challenges facing the experimental testing of claims about unobservable entities such as atoms and molecules. In Section 4, I present Perrin's early views on scientific methodology and his early arguments for the molecular hypothesis. In Section 5, I show how Perrin used the phenomenon of Brownian movement to provide a qualitative triangulation of molecular reality. In Section 6, I show how Perrin's vertical distribution experiments provided a quantitative triangulation of molecular reality. In Section 7, I show how Perrin used the multiple determination strategy (at a lower level) to defend the validity of the most doubtful (theoretical and experimental) auxiliary assumptions employed in his vertical distribution experiments. In Section 8, I identify the epistemic import of the vertical distribution experiments. In Section 9, I identify the epistemic import of the independent determinations of Avogadro's number. In Section 10, I describe the emergence of discordance after the first failed attempts to experimentally verify Einstein's mathematical theory of Brownian motion. In Section 11, I 
describe Perrin's experimental verification of Einstein's mathematical formulae and his use of the multiple determination strategy to resolve the discordance. In Section 12, I describe the reaction of the scientific community. In the concluding Section 13, I identify the elements responsible for the epistemic strength of Perrin's argument for molecular reality and hint at the development of a general conceptual framework for evaluating the epistemic strength of other concrete applications of the multiple determination strategy.

\section{The Philosophers' Stories}

Perrin's argument for molecular reality was successful in ending nearly one hundred years of scientific debates over the existence of atoms and molecules. It is not surprising, therefore, that it has been the focus of much philosophical interest. We can discern two, relatively independent, philosophical treatments.

On the one hand, Perrin is often cited in philosophical discussions over the epistemic strategy of multiple determination-i.e., the strategy of using multiple independent procedures to establish the same result (Wimsatt 1981; Hacking 1983; Kosso 1988; Culp 1994, 1995; Woodward 2006; Stegenga 2009; Soler et al. eds. 2012). ${ }^{3}$ Perrin's argument is presented as the emblematic case of multiple determination and it is used to exemplify the strategy's general rationale. More specifically, Perrin's argument is presented as a no-coincidence argument and its underlying rationale as being something along these lines: it would have been a highly improbable coincidence for thirteen different procedures to arrive at the same value for the number of molecules contained in a unit of substance and yet for molecules to not exist. Little analysis is devoted, however, to the role that the multiple determination of $N$ played in Perrin's experimental work and in convincing the scientific community. Some questions that naturally 
emerge are: Why did Perrin need to determine $N$ with multiple independent procedures (as opposed to using a single reliable procedure)? If the multiple determination strategy was so important, why did Perrin need thirteen different determinations (as opposed to only two or three)? Why did he stop at thirteen (as opposed to fourteen or twenty-three)? How did Perrin end up with thirteen different determinations for $N$ ?

On the other hand, and independently of being used as the paradigmatic case of multiple determination, Perrin's work has been the subject of more or less detailed case-studies from other philosophers of science. The aim of these studies is exactly to reveal the reasoning underlying Perrin's argument. The various philosophers, however, have arrived at different conclusions regarding both Perrin's reasoning and the role that the multiple determination of $N$ played in it. Below, I present the most important philosophical interpretations of Perrin's case.

Perrin's argument has been interpreted as an inference to the best explanation. The latter was introduced by Gilbert Harman who argued that when making such an inference "one infers, from the fact that a certain hypothesis would explain the available evidence better than any other rival hypotheses, to the truth of that hypothesis" (Harman 1965, 89). In Perrin's case, the molecular hypothesis offered the best explanation of the various experimental results.

Clark Glymour $(1975,1980)$, on the other hand, argued that Perrin's was a case of bootstrapping confirmation. In bootstrapping confirmation, hypotheses within a complex theory are used to test other hypotheses within the same theory. The bootstrapping strategy is a solution to the problem that-when experimentally testing a theory-the values of the magnitudes that can be experimentally determined often are not those, or not all of those, that appear in the theory to be tested. Bootstrapping uses the theoretical relations within the theory to be tested to compute values for the magnitudes of the theory that cannot be experimentally determined from the values 
of magnitudes that can be experimentally determined. According to Glymour, Perrin used the mathematical relations between the magnitudes appearing in the kinetic theory of gases (the theory to be tested) in order to compute a value for $N$ (a magnitude which could not be directly determined) from the values of magnitudes that could be experimentally determined (Glymour 1975, 409).

Wesley Salmon $(1978,1984)$ was one of the few philosophers who emphasized the role played by the independent determinations of $N$ in Perrin's argument. The latter, he claimed, has the structure of a conjunctive common cause argument. The reasoning underlying a common cause argument is the following: if very similar effects have been produced, and if it can be reasonably argued that the effects are causally independent (i.e., no effect causes any of the others), it can be concluded that they result from a common cause. It would be too improbable a coincidence for similar effects to have occurred independently of one another. The existence of the common cause, thus inferred, can be used to explain the effects. In Perrin's case, the agreeing values for $N$ determined by thirteen independent procedures would be an unthinkably improbable coincidence if molecules did not exist. The existence of molecules offers, in turn, a causal explanation of the agreement (Salmon 1984, 220).

Nancy Cartwright (1983), building on Salmon's 'common cause' interpretation, has offered a somewhat different analysis. Cartwright argues that the 'inference to the best theoretical explanation' interpretation misdiagnoses Perrin's reasoning. Perrin did not make an inference to the best explanation, where explanation included anything from theoretical laws to a detailed description of how the explanandum was brought about. He rather made a more restricted inference: an inference to the most probable cause (Cartwright 1983, 83). In each one of the various determinations of $N$, explains Cartwright, an experiment was used to infer facts about a 
concrete cause (molecules) from the character of its more readily observable effects (determined value for $N$ ). The reasoning from the character of the effect to the character of the cause, however, was always made against a background of other knowledge. Perrin could have been mistaken about the processes linking cause and effect in each one of the thirteen procedures: what was observed might not have resulted in the way thought from the cause under study. For example, what was observed might have been an artefact of the experimental procedure, it might have been caused by an experimental error, and so on. This is where the no-coincidence argument enters in Perrin's reasoning: Perrin's used the converge of results to argue that the various procedures used to determine $N$ were each good enough, and that they could be legitimately used to infer the cause from the nature of the effect (Cartwright 1983, 84-85). Contrary to the 'inference to the best explanation' interpretation, Cartwright argues that no theoretical laws or theoretical explanations were involved in the various determinations. Contrary to Salmon, Cartwright argues that the 'no-coincidence argument' was not about the existence of atoms and molecules as much as it was about the validity of the determination procedures-the first (Salmon's) use of the argument concerns a metaphysical issue, whereas the second concerns an epistemic issue. Cartwright's interpretation seems to downplay the necessity of the multiple determination strategy in Perrin's case: a multiple determination of $N$ would have not been necessary if Perrin could be certain about the causal connection involved in any of the various determinations (see also Cartwright 1991, 150-51).

Cartwright's interpretation has been challenged by Deborah Mayo (1986). Mayo downplays even further the role played by the multiple determination of $N$. For Mayo, an agreement among independent determinations, in and of itself, only rules out that the result is not an artifact of the determination procedures; it does not allow to pinpoint a genuine causal explanation. In Perrin's 
case, the numerical agreement only established that each one of the results was not an artifact of the determination procedure used; it could not establish that what was being determined was indeed the number of molecules in a gram-mole. Like the inference to the best (theoretical) explanation, the inference to the most probable cause suffers from a problem of underdetermination (Mayo 1986, 46). The latter, continues Mayo, can be avoided if two requirements are fulfilled. The first is to establish that the convergence of independent experimental results would be highly likely if causal hypothesis $H$ is true. In Perrin's case, one needed to show that the existence of molecules could cause the determination of $N$ in the experiments on Brownian motion, alpha particle decay, X-ray diffraction, etc. The second requirement is to establish that the convergence of independent experimental results would be highly unlikely if causal hypothesis $H$ is not true. In Perrin's case, one needed to show that other possible causes did not play a role in the various determinations of $N$. The two requirements constitute, what Mayo calls, a severe test for the hypothesis $H$. The important thing to notice is that if the two requirements are fulfilled even for one of the thirteen determinations of $N$, the multiple determination and the common cause explanation become redundant: one would have already shown that molecules are the cause behind the determination of $N$, and that no other causal factors are involved (see also Achinstein 2001, 250-51). In fact, Mayo argues that Perrin's efforts consisted exactly in providing such a severe test for the kinetic-molecular explanation of Brownian movement-and not a multiple determination of $N$. More specifically, Perrin showed how molecular motion could cause the phenomenon of Brownian movement (requirement $a$ ) and established that no other causal explanation of the phenomenon was possible (requirement $b$ ). Multiple determination could not, and did not, play the crucial role assumed by Cartwright and other philosophers (Mayo 1996, 214-50). 
Peter Achinstein (2001) has claimed that his account of evidence offers the best interpretation of Perrin's argument. According to Achinstein's account of evidence, $e$ is evidence for hypothesis $h$, given background assumptions $b$, if and only if: (a) $e$ and $b$ are true, (b) $e$ does not entail $h$, and (c) the product of the probability of $h$, given $e$ and $b$, with the probability that there is an explanatory connection between $h$ and $e$, given $h, e$ and $b$, is greater than $1 / 2$ (Achinstein 2001, 259). The last condition requires that both probabilities involved are greater than $1 / 2$. According to Achinstein, even before embarking in his experimental study of Brownian movement, Perrin believed that there was background information $(b)$ for the hypothesis $(T)$ that chemical substances are composed of molecules and that the number of molecules in a grammole of a substance is (approximately) 6.10 23 . Perrin believed, on the basis of $b$ alone, that $T$ was more probable than not (that is, the probability of $T$ given $b$ was greater than $1 / 2$ ). Achinstein then claims that Perrin's experimental work on the vertical distribution of Brownian particles found $N$ to be $6.10^{23}$. This evidence $(C)$ was not a deductive consequence of $T$. By applying Bayes' rule, Achinstein claims that $C$ made the probability of $T$ greater than it was on $b$ alone. Finally, given $T, C$, and $b$, the probability was high that there was an explanatory connection between $T$ and $C$, i.e., the probability was high that Perrin's vertical distribution experiments found $N$ to be $6.10^{23}$ because substances are composed of molecules of which the number $N$ contained in a gram-mole is $6.10^{23}$. The multiple determination of $N$ does not play an important role in Achinstein's account. The independent determinations of $N$ are used as part of the background information (b) to establish that the probability of $T$ given $b$ is greater than $1 / 2$ (Achinstein 2001, 262).

Achinstein's interpretation has been criticized by Stathis Psillos (2011a, 2011b). Psillos agrees that Perrin's reasoning is best construed as a probabilistic argument, but he claims that Perrin's experiments did more than simply increasing the probability of the atomic hypothesis 
above $1 / 2$; they made it (almost) certain. According to Psillos, Perrin applied Bayes' rule to offer an experimentum crucis for the reality of atoms.

According to Bas van Fraassen (2009), however, Perrin's experiments did not intend to, and did not establish, the reality of atoms and molecules. They only provided empirical grounding for one of the most important parameters of the kinetic theory of gases: Avogadro's number. By 'empirical grounding' van Fraassen means the process of determining definite and unique values for all the significant parameters of a theory from empirical and experimental data. Although van Fraassen's claims that empirical grounding requires that the values for a theoretical parameter determined by different means agree among them, he maintains that the agreement discloses nothing about the underlying reality upon which the parameter is grounded (van Fraassen 2009, $19-21)^{4}$

For Robert Hudson (2014), Perrin's was not a case of multiple determination, but a case of reliable process reasoning. Perrin possessed only one reliable procedure for determining $N$ : the one based on his experimental study of the vertical distribution of Brownian particles. What is usually interpreted as a multiple determination was in fact a process of calibration. Perrin used his reliable determination procedure to test and verify the other determinations of $N$.

We are thus left with a number of (mostly contradictory) philosophical interpretations about the role that the multiple determination of $N$ played in Perrin's argument. It is not difficult to locate the source of the problem. All the above philosophical approaches belong to-what has been called-the illustrative use of history. It is long known that when philosophers turn to the historical material they often begin with pre-established conceptions in mind (Richards 1992). Philosophers of science, in particular, have pre-established conceptions regarding the nature of scientific explanation, the nature of scientific theory, the nature of the relationship between 
theory and evidence, and so on, and they often use the historical material to illustrate or offer support for their pre-established conceptions. There is, therefore, the danger that the philosophers might have misunderstood, or even intentionally distorted the argumentative reasoning of the scientists they study. Ernan McMullin identified the main problems of the illustrative approach:

It makes use of the great scientists of the past as lay figures in what seems to be a historical analysis but really is not. They are manipulated to make a philosophical point which, however valid it may be in itself, was really not theirs, or at least is not really shown using the proper methods of the historian to have been theirs (McMullin 1970, 18, my italics).

In the rest of the paper, I look closely at the historical development of Perrin's reasoning and experimental work to better understand the role that the multiple determination of $N$ played in his argument for molecular reality. One important reason why the various philosophers have arrived at such different assessments is because their accounts are based on the final versions of Perrin's argument. They are usually based on the English translations of Perrin's (1909c) monograph Mouvement Brownien et Réalité Moléculaire and his (1913b) book Les Atomes. ${ }^{5}$ From these works, the philosophers cherry-pick the elements that are most beneficial to their philosophical positions, and ignore the elements that do not fit, while altogether disregarding the historical origin of these elements in Perrin's thought. In addition, and contrary to the previous philosophical efforts, I do not have a preconceived philosophical position regarding the nature of scientific confirmation, the relationship between hypotheses and empirical evidence, and so on, which I will try to illustrate, or use to interpret the historical material. ${ }^{6}$ 


\section{The Question of the Atom in the Nineteenth Century: Scientific, Philosophical, and Ideological Implications}

It is impossible to offer a detailed history of nineteenth century atomism here. Furthermore, this has been done very successfully elsewhere. ${ }^{7}$ I will, therefore, only summarize some important developments.

In the beginning of the nineteenth century, the English physicist John Dalton proposed an atomic theory, which is considered (especially its chemical part) to mark the transition from a philosophical to a scientific brand of atomism (Dalton 1908; Chalmers 2009, 173-82). Dalton claimed that his atomic theory could explain the laws by which chemical substances combined to form chemical compounds, and that it provided a way for experimentally determining the relative weight of atoms (simple or compound). Early nineteenth century chemists, however, soon realized that, in order to determine unique relative atomic weights from measurements of combining weights, one had to know the number of atoms that combined to form compound atoms (i.e., molecules). That is to say, one had to know the chemical formula of the compound. According to the traditional story, the problem of determining unique atomic formulae was solved in 1860 by Stanislao Cannizzaro, who was able to combine the Gay-Lussac law of combining volumes, from 1808, with the Avogadro hypothesis, from 1811. The former stated that when two gases combine to form other gaseous compounds, under the same conditions of pressure and temperature, the volumes of the combined gases and of the resulting compound are in whole number ratios, whereas the latter stated that, under the same conditions of pressure and temperature, equal volumes of gases contain equal numbers of "molecules". From the combination of the two hypotheses it followed that the relative molecular weights of any two gases are the same as the ratio of their densities under the same conditions of pressure and temperature. ${ }^{8}$ For historians of science who saw the fate of 
chemical atomism closely connected with the determination of relative atomic weights, the case for the chemical atom was particularly strong during the second half of the nineteenth century. Other historians, however, have challenged the view that the determination of unique atomic weights and formulae was considered, by nineteenth century chemists, to require a commitment to the real existence of atoms and molecules (Klein 2003; Needham 2004; Chalmers 2009, Chapter 9).

In addition to developments regarding chemical atomism, the second half of the nineteenth century witnessed the development of a physical atomic theory: the kinetic theory of gases. Beginning with the work of Rudolph Clausius and continuing with the work of James Clerk Maxwell and Ludwig Boltzmann, the kinetic theory made advances in explaining known empirical facts and in predicting novel phenomena about the macroscopic physical behavior of gases, based on assumptions about the motions and collisions of their constituent molecules. The kinetic theory of gases, in conjunction with other assumptions, was used to provide approximate determinations for various molecular quantities, such as Avogadro's number and the size and the velocity of the (hypothetical) molecules (Gardner 1979, 23; Chalmers 2009, Chapter 11).

Despite its early successes, the kinetic theory was perceived by many to be in decline during the time period 1880-1895. It could not generate any new predictions, and some of its fundamental assumptions were faced with experimental refutation. Two were the major problems confronting the kinetic theory at the time: (a) the experimental refutation of its predictions about the ratio of the specific heat of a gas under constant pressure to the specific heat under constant volume, and (b) the seeming incompatibility between the time reversibility of the microscopic behavior of colliding particles and the time irreversibility of macroscopic phenomena as implied by the second law of thermodynamics. ${ }^{9}$ Further, the statistical explanation of the second law of 
thermodynamics, used by proponents of the kinetic theory to explain the reversibility problem, clashed with the program of phenomenological thermodynamics which, during the same time period, was developing as a successful alternative to the atomic-kinetic program. Defined, in 1878, by Maxwell "as the investigation of the dynamical and thermal properties of bodies, deduced entirely from the first and second law of thermodynamics without any hypotheses as to the molecular constitution of bodies" (Chalmers 2009, 216) phenomenological thermodynamics developed into a successful 'research program' by the end of the nineteenth century (Clark 1976).

Thus, almost one hundred years after Dalton, and despite the obvious advances made by the atomic-molecular conception of matter, there still existed scientific disagreements regarding the existence of atoms and molecules. The atomic debates gained special importance in the fin-desiècle France where, parallel to the internal scientific and philosophical debates regarding the validity and usefulness of atomic hypotheses, there emerged a general controversy about the role and value of science in society. After two decades during which scientism and positivism had dominated the intellectual and political life of the largely anticlerical Third Republic, in the last decade of the nineteenth century, there appeared a critical reaction against science. This reaction came mainly from idealist and religious circles and expressed a general pessimism regarding the ability of science to deliver on its promise to uncover the mysteries of the universe and reveal the essential nature of things. The controversy that followed the idealistic reaction is known as the controversy over the bankruptcy of science (banquerote de la science). Although the critique was directed against physical science in general, it was the mechanistic and materialistic conception of physical and life phenomena with its scientific determinism and fatalism that was the main 
target. Within the mechanistic theories, the atomic hypotheses, seen by many as metaphysical constructions lacking empirical support, were the ones that suffered the most. ${ }^{10}$

An important argument against the ability of science to investigate unobservable entities was Pierre Duhem's famous critique of experimental method. In the early 1890s, Duhem argued that an experiment in physics is mediated by the use of instruments and theory. More specifically, an experiment in physics is not simply the observation of raw-data (concrete facts, instrument readings, etc.), but also the theoretical interpretation of these raw-data. The process of theoretical interpretation replaces the raw-data gathered from observation "with abstract and symbolic representations that correspond to them in virtue of the physical theories admitted by the observer" (Duhem 1894, 182, italics in the original). Whereas the observation of raw-data is available to the theoretically uninitiated observer, the process of theoretical interpretation requires a deep knowledge of physical theory. Thus, the trust placed on the validity of experimental results could be only as high as the trust placed on the theoretical hypotheses and auxiliary assumptions used to obtain and interpret that result. Duhem claimed that the theoretical dependence of experimental results had important implications for theory testing. When an experimental result failed to match the prediction of a theoretical hypothesis under test, it was not only the theoretical hypothesis that was at stake, but also the entire set of theoretical and other auxiliary assumptions employed to obtain the result. This critique of experimental method was a powerful argument used by Duhem against the physicists and philosophers who claimed that their experiments had arrived at microscopic explanations of macroscopic phenomena, and who appealed to the claims of positive science in order to decide what Duhem thought were metaphysical questions (Duhem 1894, 228; Coko 2015a, 75-76). 
Duhem and Perrin were both graduates of the prestigious Parisian École Normale Supérieure. But whereas Duhem was a "militant" Catholic and royalist, who was "forced" by the scientific establishment of the French Third Republic to spend all of his academic career outside Paris, Perrin was an atheist, a Marxist, and a staunch supporter of republican ideas, who held the chair

of physical chemistry at the Sorbonne for more than thirty years. ${ }^{11}$ Whereas Duhem was the chief advocate of Energetics in France and opposed until the end the atomic hypotheses, Perrin spent most of his scientific career defending the ability of science to penetrate the unobservable realm and offering evidence for molecular reality. The conceptual and historical ties between the scientific philosophies of Perrin and Duhem have never, to my knowledge, been a topic of historical investigation. As I will show in this paper, Perrin's success in establishing molecular reality consisted in understanding that what was at stake in the atomism debates was not simply the question of whether there was enough evidence to warrant belief in the existence of atoms and molecules but, even more fundamentally, whether it was necessary for physical science to postulate the existence of such unobservable entities and what could count as confirmatory evidence for their existence. In particular, the methodology underlying Perrin's experimental work can be construed as a direct response to the methodological claims of the school of Energetics and especially to Duhem's critique of the experimental method.

\section{Early Methodological Views and Molecular Hypotheses}

Jean Baptiste Perrin was born in Lille, on September 30, $1870 .{ }^{12}$ He was raised and received his early education in Lyon, before moving to Paris to enter a class of special mathematics at the Lycée Janson-de-Sailly. Studying under Émile Lacour, young Perrin was encouraged to prepare for the École Normale Supérieure. He entered the prestigious Parisian school in 1891. Perrin was 
immediately attracted to experimental physics and studied under Marcel Brillouin, one of the few French scholars who supported the kinetic theory of gases at the time. In 1895, after refusing a teaching position in secondary education, Perrin was appointed agrégé-préparateur at the École Normale. At the same time, he began his experimental work, first on cathode, and then on Röntgen rays. This early experimental work resulted in his doctorate thesis (Perrin 1897). Perrin argued that the experimental study of cathode rays suggested that they were streams of negatively charged particles (Perrin 1895; 1897, 17). Similarly, Perrin argued that some of the experimental effects produced by the Röntgen rays could be explained by the molecular framework provided by the kinetic theory of gases $(1897,46)$.

Perrin's early experimental writings did not explicitly address methodological issues. His thoughts on scientific methodology were first expressed in his book Traité de Chimie Physique: Les Principes (1903) $\cdot{ }^{13}$ In the book's preface, Perrin discusses the two fundamental methods of physical science. The first is the inductive method, which is characterized by a sure and slow march from the recording of particular empirical facts to the formulation of general principles. It is a method characterized by the defiance of all mystery and metaphysics and the disdain for everything that cannot be reduced to "effectively realizable sensations" (Perrin 1903, vii, italics in the original). Opposite the inductive method stands the deductive method, which mostly provides "explanations of the visible by the invisible." More specifically, the deductive method "consists in imagining for matter a structure, the direct perception of which still escapes our imperfect senses, and such that its knowledge would allow to deductively predict the visible properties of the universe" (Perrin 1903, vii). Contrary to the prevailing philosophical atmosphere of the fin de siècle in France, Perrin argues that, rather than being incompatible, the two methods can be fruitfully combined to investigate the properties of matter that escape 
sensory perception. This could be achieved without abandoning the inductive principle that physical science is fundamentally based on empirical facts and without falling into metaphysics. One simply had to accept the intuitive idea that what can be considered to being empirically detectable is not limited to what is currently perceived by the senses, but that it can be extended with the use of scientific instruments, the development of new observation methods, and the invention of more advanced instruments (Perrin 1903, ix; see also Perrin 1901, 449-51).

To illustrate the usefulness of the atomic-molecular hypotheses and to indicate the way in which the inductive and the deductive method could be fruitfully combined in their investigation, Perrin uses a hypothetical example inspired from the history of medicine. Thus, claims Perrin, one could have hypothesized a priori-that is, without the help of empirical evidence provided by microscopy-that contagious diseases are caused by the multiplication of very small living entities (germs). Furthermore, guided by this a priori hypothesis, one could have discovered all the Pasteurian technique and thus cured all the contagious diseases. One would have then offered an extremely useful service to humanity by using exclusively the deductive method. Yet, all these accomplishments would have been unnecessarily condemned as non-scientific by the strict inductivist, at least until the microscope could provide the necessary empirical evidence for the existence of germs (Perrin 1903, x). Perrin claimed that was enough evidence to suggest that atomic-molecular hypotheses were no different from the germ theory of disease. The atomicmolecular hypotheses had proved their legitimacy and fruitfulness by being able to deductively predict a variety of facts that were afterwards empirically confirmed. He recognized, however, that a direct experimental detection of atoms and molecules, which would transform them into real entities was still lacking. 
Les Principes was a textbook aiming to present the fundamental principles of physical chemistry. It did not contribute to expounding the existing evidence in favor of the atomicmolecular hypotheses. In an article that he had published two years earlier in the journal Revue Scientifique, and addressed to a wider readership, Perrin (1901) had presented this evidence in a detailed manner and had explained the nature of the support it provided for the molecular hypothesis. ${ }^{14}$

Some support came from the ability of the molecular hypothesis to offer simple explanations to known experimental facts. For example, it was difficult to explain the remarkable properties of crystals or why light rays of different colors propagate with different velocities in the same transparent body, without invoking the existence of molecules that gave to matter a periodically repeatable internal structure. This ability, however, offered only moderate support because it simply argued for the existence of molecules, without providing any means for detecting or measuring them.

The hypotheses regarding the molecular structure of matter made in the context of the kinetic theory of matter were of greater epistemic importance (Perrin 1901, 451-55). The kineticmolecular hypotheses not only provided explanations for the known macroscopic properties of gases and liquids, but also offered numerical approximations for the various molecular magnitudes. In addition, they helped in discovering new facts. More specifically, based on a few assumptions regarding the molecular constitution of gases and the behavior of the constituting molecules, one could explain the macroscopic physical behavior of gases as the result of the movement of the assumed molecules. By further hypothesizing on the characteristics that this molecular movement must possess in order to be able to explain the physical behavior of gases, the kinetic theory offered numerical approximations for the values of molecular magnitudes such 
as the velocity, diameter, and the number $(N)$ of molecules in a gram-mole of a substance. Thus, Maxwell's theoretical considerations on the internal friction (viscosity) of gases placed the diameter of molecules between $10^{-7}$ and $10^{-8}$, which approximated $N$ at $55.10^{22}$ (Perrin 1901 , 454). ${ }^{15}$

Perrin recognized that, in itself, this was not a conclusive argument for the existence of molecules or for the validity of the values calculated (Perrin 1901, 449). There was nothing highly remarkable about the ability of the kinetic-molecular hypotheses to explain known observable facts or provide theoretical values for the molecular magnitudes: these hypotheses were constructed in the first place exactly in order to explain and accommodate the known experimental facts. The real importance of kinetic-molecular hypotheses was their ability to derive new experimentally testable predictions. Especially, their ability to predict and explain a variety of new facts that could not be explained by other theories. One such fact was Maxwell's counterintuitive prediction that the viscosity of a gas under constant temperature ought to be independent from its density (the proximity of molecules). A prediction that was experimentally verified (Perrin 1901, 453). Perrin cited as an additional sucesesses Van der Waals application of the kinetic theory to liquids and the experimental proof that all fluids obey the same law of compressibility, independently of their chemical nature (Perrin 1901, 455). Finally, the theoretical approximations of the values for the various molecular magnitudes provided a basis to further develop and test the kinetic theory. The theoretical values could be compared with the values for the same magnitudes derived independently from the investigation of other phenomena. The atomic chemical theory, for example, was another hypothesis which was invoked to explain the empirical evidence from the chemical combination of substances. The explanation of the phenomenon of electrolysis and of the newly discovered phenomena of 
cathode and X-rays-as Perrin new firsthand from his doctoral research-had given rise to yet other hypotheses regarding the discontinuous structure of matter, although at a level deeper than that of molecules. If one could derive similar values for the molecular magnitudes from the consideration of such diverse phenomena, then one could put forward a very strong argument for molecular reality, which was the next best thing in lack of direct empirical observation (Perrin $1901,456-459){ }^{16}$

\section{Brownian Movement and the Qualitative Triangulation of Molecular Reality: The Molecular Hypothesis as a Logical Induction}

Beginning in 1901, Perrin became fully acquainted with the phenomenon of Brownian movement: the incessant and completely irregular motion of microscopic particles when suspended in a liquid (Perrin 1923, 22-28). Although it was known for the most part of the nineteenth century, it was only during the 1870 s that the importance of the phenomenon for the kinetic-molecular hypothesis was recognized (Nye 1972, 21-29; Maiocchi 1990). Perrin's main source on the topic was the work of the French physicist Léon Gouy who, at the end of the nineteenth century, had experimentally established the basic properties of the phenomenon and had demonstrated its independence from all imaginable external to the liquid influences (Gouy $1888,1889,1895)$.

Beginning in his earliest writings on the topic, Perrin argued that Brownian movement offered a different kind of evidence for molecular reality (and the probabilistic nature of Carnot's principle) from the a priori considerations made in the kinetic theory of gases (Perrin 1906a, 1906b). Whereas in the kinetic theory one postulated a priori a hypothetical molecular structure for matter from which to deductively predict observable facts, Brownian movement moved in the 
opposite direction: it provided directly observable evidence that could be used to inductively infer a molecular structure for matter. It provided a logical induction (Perrin 1906a, 96-98; 1906b, 338).

Perrin's argument was the following. The basic characteristics of Brownian movement, as established by Gouy and other nineteenth century investigators were the following: it never stopped; it was completely irregular, i.e., the movement of each suspended particle was independent from the movement of any other particle; it depended on the size of the suspended particle; it depended on the temperature of the suspending liquid; it was independent from the chemical nature of the particles; it was independent from all imaginable influences external to the liquid. These characteristics led naturally to the conclusion that the phenomenon is caused by the internal agitation of the liquid itself. There is, therefore, a continuous movement of the internal parts of the liquid (Perrin 1906b, 334-35). ${ }^{17}$ The distribution of motion in a fluid does not de-coordinate indefinitely to smaller and smaller parts, as required from the hypothesis that matter is continuous and divisible all the way down. Since the distribution of motion is limited by a spontaneous re-coordination, it follows that fluids are composed of granules that can assume all possible motions relative to one another, but in the interior of which dissemination of motion is impossible. If such granules do not exist, it is not apparent why there is a limit to the de-coordination of motion and how a phenomenon such as Brownian movement is possible. If the granules exist, however, there is an unceasingly partial re-coordination of their motion that has as a result the observable characteristics of Brownian movement. Since Brownian movement is permanent at constant temperature, the motion of the granules also has to be permanent. If these granules come into collision like billiard balls, it is necessary that they are perfectly elastic. That is, a single granule does not absorb any portion of the total motion. In summary, the 
empirical examination of Brownian movement alone was sufficient to logically suggest that every fluid is composed of elastic granules, animated by a perpetual motion (Perrin 1906b, 33536). Perrin concluded:

Now, we only need to call these granules molecules, in order to recognize an old hypothesis, glimpsed by the intuition of Epicurus and Lucretius, revived and clarified by Bernoulli, and developed by Clausius and Maxwell. Only that, this hypothesis is no longer in our eyes a priori: it ranks as a logical induction, inspired from the observation of phenomena, in the same way that, for example, the undulatory theory of light is inspired, but not imposed, by the known properties of light (Perrin 1906b, 338).

Thus, the phenomenon of Brownian movement provided an inductive argument for the existence of unobservable molecules, independent from the deductive argument provided by the kinetic theory of gases. Yet, it was an argument that fell short of establishing the kinetic-molecular explanation of Brownian movement. The weakness had to do with something that Perrin had pointed out in his 1901 paper on the discontinuity of matter: whereas one could imagine only one continuous structure for matter, a discontinuous structure could be realized in many different ways $(1901,41)$. The logical induction offered an independent argument for the claim that matter is discontinuous. It did so, however, in a purely qualitative way, which did not prove that the discontinuous parts of the liquid causing the Brownian movement were identical (or were even of the same order of magnitude) with the molecules postulated in the kinetic theory of gases. ${ }^{18}$ To establish such an identity, was required an independent quantitative triangulation. That is, one would have to use the observable properties of Brownian movement in order to calculate the values of the magnitudes causing it, and then compare the results with the values for the 
molecular magnitudes provided by the kinetic theory of gases. This is exactly the experimental path that Perrin followed, beginning in 1908.

\section{The Quantitative Triangulation of Molecular Magnitudes: The Vertical Distribution Equation and Experiments}

Perrin claimed that the best way to connect the visible characteristics of Brownian movement with the kinetic-molecular motions (supposedly) causing them was to consider the suspended Brownian particles as giant molecules-for example, similar to molecules of sugar in a solution of sugar water (Perrin 1905, 58). To establish the identity of the granular motions causing the Brownian movement with the molecular motions postulated by the kinetic theory, one had to triangulate. That is, one had to determine the mean kinetic energy of a Brownian particle and compare it with the mean kinetic energy that the kinetic theory had deduced for an isolated molecule at the same temperature. Assuming the truth of the theorem of the equipartition of energy-which stated that at the same temperature, all the molecules of all fluids have the same mean translational kinetic energy, independently of their nature (e.g., the heavy molecules of sugar moving in water have the same mean translational kinetic energy with the molecules of water)-a numerical agreement would establish that the movement of Brownian particles was caused by the motion of the liquid molecules, with the Brownian particles functioning like the (hypothetical) molecules at a level accessible to empirical investigation.

Perrin, however, did not consider this triangulation to be a crucial experiment for the validity of the kinetic-molecular explanation of Brownian movement. A numerical agreement on the values for the mean kinetic energy would indeed establish that the Brownian particles functioned as giant molecules in the way postulated in the kinetic theory. A discordant result, 
however, would not necessarily disprove the kinetic-molecular explanation. The determination of the mean kinetic energy of a Brownian particle was not a straightforward matter: it relied on many (theoretical and experimental) auxiliary assumptions. A discordant result, therefore, could be blamed on the use of faulty auxiliary assumptions.

The failure of the first experimental efforts to achieve agreement shows that this was indeed the case. The most direct way to calculate the mean kinetic energy of a Brownian particle was by measuring its size and velocity. Perrin attempted such gross calculations, but the numerical value he found was approximately 100000 times smaller than the mean kinetic energy that the kinetic theory of gases assigned to a gas or liquid molecule at the same temperature. Perrin did not, however, regard this result as a failure of the kinetic-molecular explanation (Perrin 1905, 58).

The invention of the ultramicroscope soon provided an independent explanation for the discordance. More specifically, in 1903, Richard Zsigmondy and Henri Siedentopf published in Annalen der Physik a description of the ultramicroscope, a new apparatus that could reveal details about the internal structure of matter at the limits of $5.10^{-3} \mu$ (Nye 1972,101). The advent of the ultramicroscope not only confirmed Perrin's views about the contingent nature of the limit between what is empirically detectable and what is not, but also revealed that the observed movements of Brownian particles were not their real movements: the mean velocities of the particles varied in size and direction, depending on the length of the time of observation, without ever tending to a limit.

Perrin soon found a way to determine the mean kinetic energy of a Brownian particle which did not require the measurement of its real velocity. Perrin was one of the examinateurs of Joseph Duclaux's doctoral thesis on colloid substances. There, he read about the vertical diffusion of colloid particles as a result of their irregular Brownian movement. Duclaux claimed 
that a colloid solution at a state of rest is clearer on its upper layers and denser at the bottom (Duclaux 1904, 6). Reflecting on the diffusion of colloid particles, Perrin developed the hypothesis that, if molecular motion was the cause of Brownian movement, the particles of a homogeneous emulsion (i.e., of an emulsion with particles of the same size) should, as a result of their irregular movements, distribute themselves in the same way as the (hypothetical) air molecules under the influence of gravity (Perrin 1905, 60). It was known since Pierre Laplace that the density of a gas in equilibrium decreases with altitude according to an exponential law. Further, Jacobus Van't Hoff had shown that the gas laws extend to dilute solutions. ${ }^{19}$ Under a molecular interpretation, this meant that the molecules of a solute-independently of their sizeshould behave like the molecules of a gas: their vertical distribution should also obey Laplace's exponential law. Perrin reasoned that one can legitimately hypothesize that the gas laws extend to homogenous emulsions with suspended particles large enough to be visible with an ultramicroscope. Since the suspended particles could be thought as-extremely large-assemblages of molecules, they ought to be in the same situation with the (hypothetical) molecules of the solute: they also ought to be subject to the incessant and irregular impacts from the (hypothetical) molecules of the liquid. If he could establish that the vertical distribution of Brownian particles obeyed Laplace's exponential law, he would have confirmed the hypothesis that the gas laws extend to Brownian particles and, therefore, have in the behavior of Brownian particles a magnification, in a visible scale, of the behavior of the (unobservable and hypothetical) molecules. ${ }^{20}$ An experimental determination of the osmotic pressure exerted by a Brownian particle would then be possible. This experimental value would be compared with the value that the kinetic theory of gases (based on different assumptions and reasoning processes) had theoretically deduced for the pressure exerted by a single molecule, under similar conditions. 
A numerical agreement would then provide the much-needed quantitative confirmation of the kinetic-molecular explanation of Brownian movement.

Perrin attempted the experimental determination of the osmotic pressure of a Brownian particle in early 1908. He published the results in the Comptes Rendus de l'Académie des Sciences. His goal was to prove "that molecular agitation is an actual cause, and cause unique, of Brownian movement" (Perrin 1908a, 968). ${ }^{21}$ By diluting a stick of gamboge (a gum resin) in water, Perrin created a yellow emulsion where a lively Brownian movement of gamboge particles could be perceived with an ordinary microscope. Perrin used fractional centrifuging to obtain a homogenous emulsion (Perrin 1909c, 36-38). The remaining Brownian particles could only be observed with the lateral illumination of the ultramicroscope. The particles had the same brightness, which Perrin interpreted as further indicating that they had the same size, and did not twinkle, which Perrin interpreted as indicating that they were of spherical shape. $49,586 \mathrm{~cm}^{3}$ of the emulsion contained 49,299 $\mathrm{g}$ of water and $0,287 \mathrm{~g}$ of gamboge, which gave a density of 1,35 for the granules studied. To study the vertical distribution of the particles, Perrin placed a drop of this emulsion in a microscopic preparation. After waiting several hours for a state of equilibrium to be attained, he counted the number of granules that could be observed at different heights. ${ }^{22}$ After doing thousands of such readings and taking their average, Perrin found that the equilibrium vertical distribution of the granules in the microscopic preparation (and probably in all colloidal solutions) is indeed "exponential, like that of a gas under the influence of gravity" (Perrin 1908a, 969). He then argued that that the exponential vertical distribution could be explained in a way that allowed the calculation of the osmotic pressure of a single Brownian particle. More specifically, identical particles of density $\rho$, and mass $m$, would exert with their impacts, on the walls of a membrane stopping them but allowing the water molecules to pass 
through, an osmotic pressure proportional to their concentration $k n$ (where $k$ is the osmotic pressure exerted by a single granule and $n$ the number of granules in a unit of volume). If $n d h$ is the number of granules contained in a horizontal slice of height $d h$ and unit cross-sectional area, then they would be maintained in suspension by the sum of the upward buoyant force and the difference between the osmotic pressures exerted on the two faces of the slice $d h$. This allowed the derivation of the equation:

$$
\frac{d n}{n}=\frac{1}{k} g d h\left(1-\frac{1}{\rho}\right) m
$$

Which, integrated between levels 0 and $h$, became

$$
2,3 \log \frac{n_{0}}{n}=\frac{1}{k} m g h\left(1-\frac{1}{\rho}\right)
$$

This is the original version of Perrin's vertical distribution equation which allowed the determination of $k$, if one could determine $m$ and $\rho$.

To determine $m$, Perrin used Stokes' law, which states that the force of friction resisting the movement of a sphere in a viscous medium at each instant is equal to $6 \pi \mu \alpha v$ (where $\mu$ is the viscosity of the medium, $\alpha$ the radius of the sphere, and $v$ its velocity). When the sphere falls with uniform velocity only under the influence of gravity, the force of friction ought to be equal to the apparent weight of the sphere in the fluid. That is:

$$
\frac{4}{3} \pi \alpha^{3} g(D-d)=6 \pi \mu \alpha v
$$

Where $D$ and $d$ are the densities of the sphere and the fluid, respectively. The equation gives $\alpha$ if the speed of fall is measured. Perrin applied this relation to the speed of fall of the Brownian particles in the upper layer of an emulsion that had not yet reached a state of equilibrium. Using a capillary tube to avoid convective movements, Perrin measured a fall of $0,97 \mathrm{~mm}$ per day 
(Perrin 1909c, 44-46). The resulting value for $m$ was $9,80.10^{-15}$. Substituted in the vertical distribution equation, it gave for the osmotic pressure exerted by a Brownian particle $(k)$ the value $360.10^{-16}$. Perrin compared the osmotic pressure exerted by $n$ Brownian particles with the pressure that, according to the kinetic theory, was exerted by $n$ molecules of a gas at the same temperature. This pressure would be equal to $n \frac{R T}{N}$ (with $R$ being the constant of perfect gases, $T$ the absolute temperature, and $N$ the number of molecules contained in one gram-mole, which the considerations from the viscosity of gases, according to Perrin, placed it at $7.10^{23}$. By doing the calculations, Perrin found this pressure to be equal to $n \times 343.10^{-16}$. Almost equal to the osmotic pressure of $n$ Brownian particles, assuming the validity of the value for $N$. Therefore, concluded Perrin, the suspended Brownian particles functioned like visible molecules of a perfect gas with a molecular weight of around three billion. The conclusion of Perrin's first 1908 experimental paper reads:

The mean kinetic energy of a colloid particle is therefore equal to that of a molecule. Thus, it is established by experiment, the hypothesis which Einstein and Langevin have pointed out as equivalent to that of M. Gouy (theorem of equipartition of the kinetic energies). At the same time, the kinetic theory of fluids seems a little more fortified, and the molecules a little more tangible. Their number $N$ in a gram-mole, deduced from the previous equality, assumed to be correct, is $6,7.10^{23}$ (Perrin 1908a, 970, italics in the original).

Perrin used the quantitative agreement between the experimental value for the osmotic pressure of a Brownian particle and the theoretical value for the pressure of a single molecule under the same conditions, to argue not only for the validity of the determined value, but also about the validity of the central theoretical and experimental auxiliary 
assumptions underlying his experimental approach. Especially for the validity of the theorem of the equipartition of energy and the claim that molecular motion is indeed the (unique) cause of Brownian movement. The underlying reasoning was that-since the validity of the auxiliary assumptions used to determine the value for $k$ did not depend on the validity of the assumptions used in the kinetic theory of gases to determine the pressure of a molecule under the same conditions-the auxiliary assumptions would have to be flawed in a very remarkable way for them to arrive at such improbable numerical agreement. It was difficult to explain this numerical agreement if one did not accept the conclusion that Brownian particles functioned as giant molecules in the way postulated by the kinetic theory.

\section{Duclaux's Critique and Other Experimental Work from 1908}

Perrin's conclusion-based as it was on a strong no-coincidence argument from the almost absolute agreement on the numerical values for a molecular magnitude determined by independent procedures-seemed almost unassailable. In reality, however, it was challenged immediately. Joseph Duclaux, whose work was crucial for connecting the vertical distribution of Brownian particles with the kinetic theory of gases, raised several objections against Perrin's experiments (Duclaux 1908). Duclaux criticized several aspects of Perrin's experimental work: the determination of the density of the particles, the determination of the mass of the particles, and the determination of the $\frac{n}{n_{0}}$ ratio. First, Duclaux pointed out that gamboge was soluble in water and, therefore, in the emulsion that Perrin had prepared part of the substance must have been in a state of real solution. This must have influenced Perrin's values for the density of the gamboge particles. Second, the fact that the particles appeared to have the same size under the 
lateral illumination of the ultramicroscope was not sufficient to prove that they were of equal size. Third, and most importantly, Duclaux claimed that Perrin's application of Stokes' law to the free fall movement of the particles in order to calculate their mass "seemed extremely risky," because the properties of Brownian movement were different from the conditions of continuity required by the law. Moreover, the speed of the fall that was used to calculate the radius had nothing to do with the true velocity of the particles with respect to the fluid, which was incomparably greater and continuously changing in magnitude and direction. In addition, Duclaux argued that Perrin's theoretical reasoning seemed very simplistic. It involved, for example, the derivative $\frac{d n}{d h}$ of the concentration expressed as a function of height. For Duclaux, this concentration could not be treated without a justification as a definite and continuous function at each point, especially given that the position of every particle was governed by the laws of probability (Duclaux 1908, 132-33).

Duclaux's critique, however, was directed towards the degree (or quality) of the quantitative agreement which Perrin claimed to have achieved, and not against the existence of some sort of agreement (Duclaux 1908, 133). Duclaux's position was that molecular motion, as postulated by the kinetic theory, was indeed a cause of Brownian movement-which could explain a rough numerical agreement. But he believed that it was not the unique cause-which required that the numerical agreement was not absolute. ${ }^{23}$ Duclaux, therefore, did not have to argue against the thrust of Perrin's no-coincidence argument. That is, he did not have to explain how it was possible for Perrin's experimental approach to arrive at such a remarkable agreement with the value for molecular pressure derived independently in the kinetic theory of gases, despite it being based, as Duclaux claimed, on flawed experimental auxiliary assumptions and doubtful theoretical reasoning. 
Perrin took Duclaux's criticism seriously, and his next three experimental papers tackled the objections raised. His next experimental paper defended the use of Stokes' law for determining the radius of Brownian particles (Perrin 1908b). Perrin was able to determine the radius of Brownian particles by two other experimental procedures that were independent of Stokes' law. The first procedure made use of the fact that in a feebly acid medium the gamboge particles became affixed to the walls of the glass containing the emulsion. This allowed Perrin to count the number of particles contained in a known, previously titrated, volume of emulsion. In turn, this allowed the determination of the mass of the particle and, since the density was known, also of the radius. Further, Perrin observed that sometimes the gamboge particles arranged themselves side by side in the walls of the glass, forming rectilinear rods composed of 5 to 6 granules. The length of these rods could be measured in a camera lucida. This gave him a third, independent from the other two, procedure for determining the radius. Perrin applied all three procedures to determine the radius of Brownian particles in four different preparations, each one made up of particles of different size. The three procedures gave concordant results (Perrin 1908b, 476). Perrin used the numerical concordance obtained by the three mutually independent procedures to argue, not only about the correctness of the result but, foremost, about the validity of the procedures and their underlying auxiliary assumptions. ${ }^{24}$ Regarding Stokes' law, in particular, Perrin concluded that "it is therefore certainly valid for radii of the order of a tenth of a micron, and it is safe to assume that it is valid even beyond that" (Perrin 1908b, 476).

Perrin's third experimental paper from 1908 also contained results of experimental research that was done in response to Duclaux's objections (Perrin 1908c). This time Perrin had to correct his initial value for the osmotic pressure in light of Duclaux's criticisms regarding his calculation of the density of the particles. After all the corrections were made, Perrin replaced the initial 
value $36.10^{-15}$ found in his first series of experiments with the corrected value $42,5.10^{-15}$, which gave for $N$ the value $5,7.10^{23}$. In his 1909 monograph, Perrin describes in detail how he was able to remove the doubts surrounding the determination of the density of Brownian particles (Perrin 1909c, 38-40). Once again, he used two independent procedures that gave concordant numerical results. In the first procedure, Perrin heated the mass of resin present in a sample of emulsion. Once the temperature was raised above $130^{\circ} \mathrm{C}$, the resin turned into a viscous fluid that on cooling gave a transparent glass. Perrin assumed that the glass had the same density as that of the granules of the emulsion. Its density was easily determined by putting fragments from it in water, and adding potassium bromide until the fragments remained suspended, neither rising or falling (Perrin 1909c, 29). In the second method, Perrin measured the masses $m$ of water and $m^{\prime}$ of the gamboge emulsion that at a temperature of $20^{\circ} \mathrm{C}$ filled the same specific gravity flask. The mass $\mu$ of gamboge contained in the mass $m^{\prime}$ of emulsion was determined by drying, giving the mass of the intergranular water $\left(m^{\prime}-\mu\right)$. If $d$ is the absolute density of the water, $\frac{m}{d}$ gives the volume of the flask, $\frac{m^{\prime}-\mu}{d}$ gives the volume of the intergranular water, and their difference $\left[\frac{m}{d}-\frac{m^{\prime}-\mu}{d}\right]$ gives the volume of the granules. The density of the particles could then be found by dividing their mass $\mu$ by their volume. The two methods gave concordant numerical results when applied to determine the density of the granules of emulsions of mastic and gamboge. Once again, Perrin used the numerical concordance obtained by independent procedures to argue for the validity of the procedures and their underlying auxiliary assumptions (Perrin 1909c, 39-40).

Despite having to adjust his initial experimental value for $k$ (and consequently for $N$ ), Perrin argued that there was still an extremely striking agreement (l'accord est donc extrêmement frappant) with the theoretical value independently determined in the kinetic theory of gases (Perrin 1908c, 531). The numerical agreement gave rise to a no-coincidence argument, which 
was used to argue both about the correctness of the value determined and the validity of the (theoretical and experimental) auxiliary assumptions used in the independent determinations. In fact, as already mentioned, Perrin's aim was not to determine the osmotic pressure of a Brownian particle per se. He used the numerical agreement to argue about the validity of the many auxiliary assumptions employed in his experimental approach. In particular, the validity of the assumption that Brownian particles did indeed function as giant molecules. For Perrin, the striking numerical agreement left no doubt about the molecular-kinetic explanation of Brownian movement. This is supported by the way Perrin concludes his third 1908 experimental paper: This agreement cannot leave any doubt about the rigorous exactness of the kinetic theory of Brownian movement. But a new result is obtained thenceforth in addition: by repeating as carefully as possible an experiment analogous to the previous ones, one can determine, with a precision that I believe is hitherto unknown, the universal constant $N$ and other constants that depend on it, among them the charge of the atom or corpuscle of electricity (Perrin 1908c, 532, my italics).

That is to say, only after establishing the validity of the auxiliary assumptions employed in the experimental determination of $k$, Perrin's goal became that of providing a precise numerical value for $N$. The choice of $N$ was justified by it being a universal constant and by its direct connectedness with other molecular constants. As Perrin explained in the beginning of his fourth 1908 experimental paper:

The number $N$ of molecules contained in all gram-moles, the charge $e$ of electron and the quotient $\alpha$ of the mean energy of a molecule to its absolute temperature $T$ 
are universal constants which can be known once one of them is known (Perrin 1908d, 594).

The multi-connectedness of $N$ allowed for additional independent determinations of its value from experimental determinations of the values for $e$ and $\alpha$. By October 1908, Perrin had conducted three series of experiments using Brownian particles of different sizes. They involved calculations for 13000 particles and 16000 readings. Their average gave for $N$ the value $71.10^{22}$ (Perrin 1908d, 595).

\section{The Epistemic Import of the Vertical Distribution Experiments}

In Section 2, I showed how different philosophers, starting from different pre-conceived philosophical positions, have offered different interpretations of Perrin's experimental work. In Sections 3 to 7, by following the historicist approach, I placed Perrin's experiments on the vertical distribution of Brownian particles in their temporal dimension. Having done so, we are now able to tackle the important question: What was the structure and epistemic import of Perrin's vertical distribution experiments? The historicist approach shows a number of things:

1. The vertical distribution experiments were part of a case of multiple determination (or triangulation). After the publication of the four short research papers in the journal Comptes Rendus of the Académie des Sciences, Perrin published a longer article in the journal La Revue du Mois, where he presented the results of his experimental work to a wider readership. Writing for a wider readership, Perrin was more explicit regarding the methodology underlying, and the epistemic import of, the vertical distribution experiments. Perrin argued that the vertical distribution experiments offered an independent experimental determination for the values of molecular magnitudes that were 
first determined theoretically in the kinetic theory of gases. Perrin presented his work as a continuation of his early methodological views. As in Les Principes, he began by presenting the essential characteristics of inductive and deductive reasoning-the two faculties that constitute the essential qualities of a good physicist (Perrin 1908e, 513). Perrin argued that both forms of reasoning, independently of one another, had arrived at the conclusion that matter has a molecular structure. He reminded his readers that in an article published two years earlier in the same journal (see Section 5 above) he had shown how both deductive reasoning from the kinetic theory and inductive reasoning from observations of the phenomenon of Brownian movement had led to this same conclusion. Now Perrin wanted to present to his readers what was lacking from the earlier article: a precise quantitative agreement between the numerical values for the molecular magnitudes calculated from the theoretical considerations in the kinetic theory of gases with those obtained by the experimental investigation of Brownian movement. ${ }^{25}$ The quantitative agreement between the two determinations established that the molecules, hypothesized in the kinetic theory of gases, were real and that they were identical with the 'granules' that based on inductive reasoning from empirical observations ought to be the cause of the phenomenon of Brownian movement (Perrin 1908e, 514-15).

2. The two determinations were theoretically independent. They were based on different reasoning processes (inductive vs. deductive), on the consideration of different phenomena (Brownian movement vs. viscosity of gases) and, most importantly, on theoretically independent auxiliary assumptions. ${ }^{26}$ 
3. The numerical agreement achieved was extremely striking, especially if one considered the possible numerical values for $k$ that could have been the result of the vertical distribution experiments. According to Perrin, the range of possible experimental values for $k$ extended from zero to infinity (Perrin 1908c, 531).

4. Perrin used the agreement between the numerical value for $k$ obtained in his vertical distribution experiments with the value for molecular pressure deduced in the kinetic theory of gases to argue, not only about the validity of the result, but-perhaps even more importantly-about the validity of the central theoretical and experimental auxiliary assumptions upon which his vertical distribution experiments were based (Perrin 1908e, 528). The theoretical auxiliary assumptions included: the theorem of the equipartition of energy (which was central for the claim that Brownian particles functioned just like the molecules postulated by the kinetic theory of gases), the claim that the laws of perfect gases extended to uniform emulsions (with the particles of a uniform dilute emulsion behaving like the molecules of a gas or liquid in equilibrium), the extension of Stokes' law to the order of magnitude of Brownian movement, and the claim that molecular movement was the (unique) cause of Brownian movement. The numerical concordance was also used to argue about the validity of the experimental assumptions and procedures employed to measure the magnitudes that appeared in the vertical distribution equation. These included the procedures used: to prepare a uniform emulsion with spherical granules of equal diameter, to calculate the mass of the granules, and to determine the ratio $\frac{n_{0}}{n} \cdot{ }^{27}$ Perrin's underlying reasoning was that it would be a remarkable coincidence for independent determinations to arrive at such a striking numerical agreement on the values of the molecular magnitudes measured, and yet for the (theoretical and 
experimental) auxiliary assumptions underlying them to be essentially flawed. This form of reasoning is crucial. Because of the large number and the (somewhat) precarious nature of the auxiliary assumptions required to determine the magnitudes appearing in the vertical distribution equation, no theoretical or experimental determination, by itself, could ever be sufficient to establish both the validity of the result and the validity of the determination procedure. Only the strong no-coincidence argument that emerged when independently theory-dependent procedures converged on the same value for $k$ and molecular pressure could be used to argue both for the validity of the result and the validity of the determination procedures.

Moreover, when the degree of the numerical agreement achieved was challenged, Perrin employed the same strategy of multiple determination at a lower level to establish the validity of the auxiliary assumptions being challenged. Thus, he was able to justify his use of Stokes' law to measure the radius of the particles by obtaining concordant numerical results with experimental procedures that relied on independent auxiliary assumptions. He followed the same strategy to defend the experimental procedure used to measure the density of Brownian particles. In all cases, the underlying rationale was that it would very implausible for procedures based on independent auxiliary assumptions to provide such numerical agreement on the values of the magnitudes being measured, if their underlying auxiliary assumptions were essentially flawed.

Perrin's use of independent determinations to argue about the validity of the auxiliary assumptions makes sense if we consider that the most influential critique against the ability of the experimental method to establish the validity of claims regarding the unobservable realm, at the time, was Pierre Duhem's thesis that an experiment in 
physics involves the use of auxiliary assumptions which are practically impossible to test in isolation (Duhem 1894). ${ }^{28}$ Duhem had, in addition, claimed that not only the experimental method cannot provide access to the unobservable realm, but also that such access was not even useful. Physical theory, according to (Duhem1893; 1906), is a classification of experimental laws revealing the relations holding among the observable parts of reality, and any attempts to investigate the nature of the unobservable was akin to mixing physics and metaphysics. Although Duhem's name is not mentioned, the last paragraph of Perrin's (1908e) article in La Revue du Mois is an explicit response to the reader holding the Duhemian position. ${ }^{29}$

5. Perrin claimed that one important feature of the vertical distribution experiments, which distinguished them from other efforts of determining the molecular magnitudes, was that they allowed an unlimited precision in determining the values for $k$ and $N$. As Perrin would constantly repeat in his writings, providing a precise value for $N$ from the vertical distribution experiments was simply a question of conducting very careful experiments and making precise measurements of the magnitudes appearing in the vertical distribution equation. $^{30}$

\section{The Epistemic Import of the Independent Determinations of Avogadro's Number}

To recapitulate, after his initial experiments on the vertical distribution of Brownian particles Perrin claimed that the numerical agreement he had achieved: (a) validated his experimental approach, (b) established without a doubt the kinetic-molecular explanation of Brownian movement, and (c) provided a first determination of various molecular magnitudes. As already shown, (at the end of Section 7) it is only after this initial experimental work that providing a 
precise value for $N$ became central to Perrin's experimental work. $N$, because of its direct connection with other molecular magnitudes served as some sort of common ground to coordinate between the various determinations of molecular magnitudes that were emerging from the study of different phenomena (Perrin 1908e, 529, 532). More specifically, at the time when Perrin concluded his vertical distribution experiments (end of 1908), besides the value for $N$ calculated from the theoretical considerations made in the context of the kinetic theory of gases, there were available four other determinations. They all (largely) agreed with Perrin's. Determinations of the charge of the electron $(e)$, which were conducted at the Cavendish laboratory, placed $N$ between $43.10^{22}$ and $96.10^{22}$. Max Planck's and H.A. Lorentz's calculations, which were based on the theory of black-body radiation, gave for $N$ the values $61.10^{22}$ and $77.10^{22}$, respectively. Finally, Rutherford's calculations of $e$, which were based on the study of radioactivity, placed $N$ between $62.10^{22}$ and $77 \cdot 10^{22}$. To these values Perrin added his own value of $71.10^{22}$ by "a method which seems to me direct and susceptible to an unlimited precision" (Perrin 1908d, 595, italics in the original).

In Section 2, I showed how the various philosophers who have used Perrin's work as a case study disagree not only regarding the structure of his argument for molecular reality, but also regarding the role that the independent determinations of $N$ played in it. The historicist approach followed here allows us to tackle this issue conclusively. To recapitulate, the question we are faced with is the following: What did Perrin think was the importance of these additional determinations of $N$ ? This is especially given that Perrin maintained that his experimental work had already established both the kinetic-molecular explanation of Brownian movement and the validity of the value determined for $N$. By reading Perrin's writings from this period, we can infer several reasons why he thought the additional determinations were important. 
1. The Lack of Discordant Results. Given that Perrin thought he had already established the validity of his experimental approach and was certain that it could be used to provide precise values for $N$, the fact that it was not contradicted by independent determinations was a relief. Discordant results could have raised doubts about the molecular theory of Brownian movement and required further experimental investigation to determine the source of discordance. In fact, this is exactly what happened when the first experimental efforts to verify Einstein's mathematical theory on the molecular origin of Brownian movement led to results that were discordant with Perrin's (see next Section). The lack of discordant results was extremely striking if one considered the a priori possibilities for the values for $N$ that were possible in each one of the different determinations.

2. The Variety of the Phenomena Investigated. The different determinations were based on the theoretical consideration and the experimental investigation of different phenomena: viscosity of gases, Brownian movement, black body radiation, radioactivity, and the electric charge of ions. This variety of phenomena not only provided the required theoretical independence of the different determinations but was to be expected (one could say that it was even required) given that one was trying to determine the value of a fundamental magnitude concerning the "building blocks" of observable phenomena (Perrin 1909a, 38-39).

3. The Genetic Independence of the Determinations. Besides being theoretically independent, the different determinations were also genetically independent. What is meant by 'genetically independent' is simply the fact that the different determinations were conducted independently of one another. ${ }^{31}$ Although Perrin did not use the term, he was fully aware of the possible objection that the achieved agreement could be construed 
as a case of experimental calibration or mutual adjustment of the experimental results.

Perrin explicitly stated how fortunate he was that some of the experimental

determinations of $N$ were conducted concurrently with his, without the different

researchers having knowledge of each other's results. This precluded the possibility that

they had calibrated (deliberately or even subconsciously) their experiments to achieve agreement (Perrin 1909c, 108).

4. The Offering of Mutual Support. The theoretical independence of the determinations, the genetic independence of the determinations, the number of determinations, the quantitative nature of the agreement, the variety of phenomena considered, the lack of discordant results, were used to construe a strong no-coincidence argument to support the validity of the auxiliary assumptions underlying the different determinations, especially those based on the investigation of new phenomena (like black-body radiation and radioactivity) and which were thought to be based on untested and more speculative auxiliary assumptions. The argument's rationale was that it would be a highly improbable coincidence for the various determinations to arrive at the same value for $N$, independently of one another, and yet for the auxiliary assumptions underlying them to be essentially flawed.

\section{Mathematical Theories of Brownian Movement and the Emergence of Discordance}

Perrin's experimental work on Brownian movement did not stop with the vertical distribution experiments. Perrin was able to provide two additional concordant values for $N$ via his experimental study of the mean horizontal displacement and mean rotation of Brownian particles. What did Perrin think was the importance of these additional determinations? Looking 
at the events from a historical perspective-as opposed to only looking at the final form of Perrin's argument-shows that Perrin's aim in conducting this additional research was not to offer yet another experimental determination for $N$, but to remove the doubts about the kineticmolecular explanation of Brownian movement that emerged when the first efforts to experimentally verify Albert Einstein's mathematical work on Brownian motion failed to do so.

In 1905, without knowing that the phenomenon of Brownian movement had already been observed and studied for around eighty years, Einstein produced a mathematical formula describing the average horizontal displacement that, according to the kinetic theory of heat, the (hypothetical) kinetic-molecular motions in a liquid ought to be causing on microscopic particles suspended in it (Schilpp ed. 1949, 47). ${ }^{32}$

$$
\lambda_{x}=\sqrt{t} \sqrt{\frac{R T}{N} \frac{1}{3 \pi k P}}
$$

Where $\lambda_{x}$ is the mean horizontal displacement of a suspended (Brownian) particle, $t$ is the time interval during which the displacement is measured, $T$ is the absolute temperature, $N$ is Avogadro's number, $k$ is the coefficient of viscosity of the liquid, and $P$ is the radius of the particle (Einstein 1956a, 18).

The important thing about Einstein's formula was that it defined the horizontal displacement of a suspended particle without involving its real velocity, which could not be calculated because of the extremely complicated path the particle ought to be describing during a specific time interval. This opened the way for an experimental confirmation, since all the other magnitudes could (theoretically at least) be experimentally determined. Einstein concluded his 1905 paper by hoping "that some enquirer may succeed shortly in solving the problem suggested here" (Einstein 1956a, 18). 
The experimental testing of the displacement formula, however, was not a straightforward issue. On the one hand, assuming that the formula was correct, and that it was relatively unproblematic to experimentally determine the radius of the suspended particles and the viscosity of the intergranular liquid, one still had to assume a value for $N$ in order to provide a prediction for the mean displacement. That is, the precision of the prediction depended primarily on the exactness of the numerical value attributed to $N$. On the other hand, one could attempt to experimentally determine the radius, the viscosity, and the mean displacement and thus provide a a value for $N$. In the latter case, however, besides the validity of the formula, one ought to assume the validity of the determination procedures employed to determine the various magnitudes, especially the validity of the procedures employed to determine the mean displacement.

The difficulties surrounding the experimental testing of Einstein's formula became apparent from the very first attempts. The publication of Einstein's displacement formula was followed by three verification attempts. They were by The Svedberg in Sweden, Max Seddig in Germany, and Victor Henri in France. They all, and independently from one another, failed to verify the formula. Svedberg argued that his experimental results offered a rough verification of the formula, but his claims were rejected by his contemporaries, including Einstein, Perrin, and Paul Langevin (Kerker 1976). ${ }^{33}$ Seddig accepted the failure, but blamed the experimental procedure that he employed to determine the mean displacement (Nye 1972, 125).

The experimental failure that had the most impact on Perrin and on the French scientific community at the time came from the cinematographic study of Brownian movement undertaken by Victor Henri, at the College de France, in the beginning of 1908 (Henri 1908). Henri took cinematographic images of spherical particles of a rubber latex-apparently of uniform size of 
about $1 \mu$ in diameter-suspended in water. The cinematography used provided 20 images per second, with the duration of exposure being $1 / 320$ of a second. By determining the position of a specific particle in a successive series of photographs, Henri was able to draw the projection of the path described. He found that the trajectory described by a specific particle was extremely complex and entirely independent from the trajectories described by the other particles. The experimental value for the mean displacement during the time interval of $1 / 20$ of a second was $0.62 \mu$ and varied very little from one particle to another. This experimental value was almost four time higher than the $0,16 \mu$ value calculated by substituting in Einstein's displacement formula the values for the viscosity of water, the time interval, and the radius of the particles used in the experiment and taking $N$ equal to $7.10^{23}$. Henri concluded that Einstein's displacement formula did not apply to the Brownian movement of the particles that he had experimentally studied. Trying to locate the source of discordance within Einstein's theoretical reasoning, Henri questioned the legitimacy of applying Stokes' law to the mathematical analysis of the movement of Brownian particles of around $1 \mu$ in diameter (Henri 1908, 1026).

Following the publication of Henri's experimental results, the French physicist Aimé Cotton published, in June 1908, in La Revue du Mois an article where he addressed the discordance between Henri's experimental results with both the theoretical values predicted in Einstein's formula and the results of Perrin's experimental study of the vertical distribution of Brownian particles (Cotton 1908). Trying to locate the source of discordance, Cotton wondered whether the great quantity of light that was necessary for the photographic recording-without changing the registered mean temperature of the emulsion-had some effect on the mean displacement values measured. Cotton suggested for Henri to test this hypothesis by increasing the intensity of the illuminating beam and investigating whether the mean displacement values remained invariant. 
The other plausible source of discordance was the application of Stokes' law to particles of microscopic size. To test the legitimacy of this application, Cotton suggested for Henri to extend his cinematographic studies to other liquids and particles of different size and to investigate whether the movement of these particles in various viscous medium's obeyed Stokes' law (Cotton 1908, 740). Cotton admitted that, if correct, Henri's experimental results had failed to establish molecular motion as the unique cause of Brownian movement. Henri's results were consistent with the position-still defended by many French physicists at the time-that the electric actions exerted from the ions of the liquid on the electrically charged suspended particles were an additional cause of the phenomenon (Cotton 1908, 739; Duclaux 1908, 133). This conclusion was in conflict with Perrin's results from the investigation of the vertical distribution of Brownian particles. Cotton suggested for Perrin to control the experimental procedure he had used to determine the mass of the Brownian particles by applying it to particles larger in size and of well-known dimensions. Cotton concluded that the ideal would be for Henri and Perrin to apply their different experimental procedures on the same emulsion (Cotton 1908, 741).

\section{Perrin's Experimental Verification of Einstein's Formulae}

The situation that emerged after Henri's experiments was perplexing and called for an explanation (Perrin 1909a, 32). Einstein's theoretical investigations on the displacement of microscopic particles suspended in liquids were done independently of any knowledge of the phenomenon of Brownian movement. The very existence of Brownian movement as an observable phenomenon with the qualitative characteristics described by Einstein, spoke in favor of Einstein's theory. The displacement formula was independently derived by the Polish mathematician Marian Smoluchowski (Nye 1972, 118-21) and-by following a different 
mathematical approach-by Perrin's friend Paul Langevin (1908). Further, Perrin had demonstrated, by extensive use of the multiple determination strategy, the legitimacy of the auxiliary assumption that was most challenged in the derivation of Einstein's displacement formula: the application of Stokes' law to the order of magnitude and to the discontinuous conditions characteristic of Brownian movement. Finally, Svedberg had shown the independence of Brownian movement from the electric charge of the suspended particles (Kerker 1976, 201), showing thus the inadequacy of the only viable alternative causal explanation of Brownian movement.

Perrin believed that the vertical distribution experiments had established beyond any reasonable doubt the kinetic-molecular explanation of Brownian movement. The failure to experimentally verify Einstein's displacement formula, however, put in front of him a choice between the insufficiency of the kinetic-molecular explanation and the inexactness of the formula. Perrin chose the latter option, believing that some unjustified assumption had entered in Einstein's reasoning. Nevertheless, under the influence of the suggestions made by Cotton and Langevin, he attempted a verification of the displacement formula with the same Brownian particles of exactly known radius that he had used in his vertical distribution experiments (Perrin 1909a, 32).

The first displacement measurements were conducted by Chaudesaigues, a doctoral student working in Perrin's laboratory (Chaudesaigues 1908). Chaudesaigues reasoned that since the vertical distribution experiments had demonstrated the validity of the kinetic-molecular explanation of Brownian movement, Henri's failed attempts to experimentally verify Einstein's formula meant either (a) that some of the complementary auxiliary hypotheses used by Einstein were incomplete or inexact, or (b) that some unknown experimental complication had falsified 
Henri's attempts. An experimental test of the formula by using Brownian particles of exactly known radius could help decide the dilemma (Chaudesaigues 1908, 1045). Chaudesaigues used emulsions with spherical particles of gamboge of exactly known radius which Perrin had used in his more precise series of the vertical distribution experiments. The experiment consisted in dotting the position of a particle in a camera lucida after time intervals of 30, 60, 90, and 120 seconds. Chaudesaigues made hundreds of such displacement measurement in several series of experiments in which he varied the radius of the particles and the viscosity of the intergranular liquid. Substituting in the displacement formula the values of the parameters used in the experiments and the value determined for the mean square displacement of a Brownian particle, he found for $N$ the value $64.10^{22}$, which was "in satisfactory agreement" with the value 70,5.10 22 found in Perrin's experimental study of the vertical distribution of the same particles. Chaudesaigues used the numerical agreement to support the theoretical auxiliary assumptions employed in Einstein's derivation. He concluded that “Einstein's formula should be considered to be exact," implying that some unknown complication was the reason behind Henri's failed verification attempts (Chaudesaigues 1908, 1045).

After Chaudesaigues' initial success, Perrin repeated the experiments with the help of another doctoral student. Perrin varied even further the parameters of the experiment: the nature, diameter, and density of the particles, and the nature of the intergranular liquid (Perrin and Dabrowski 1909). In 1909, Perrin announced a mean value for $N$, calculated by around 3000 displacement recordings, equal to $71,5.10^{22}$. This value was almost identical with the value for $N$ determined in the vertical distribution experiments, and it remained relatively invariant to changes of the various experimental parameters. Perrin used the numerical agreement to support the validity of both the experimental procedures employed to determine the magnitudes 
appearing in Einstein's formula and the theoretical assumptions underlying Einstein's mathematical derivation. He concluded that "the triumph of the kinetic theory, giving the same number by routes so different, is indisputable" (Perrin 1909b, 33).

After the verification of Einstein's displacement formula, Perrin saw the possibility of an experimental test of Einstein's mathematical formula for the rotational Brownian movement. Einstein had theoretically demonstrated that the molecular impacts, besides a translational movement, impart on the suspended microscopic particles also a rotational movement (Einstein 1956b, 33). Assuming that the theory of equipartition of energy-which claimed that, at the same temperature, the mean kinetic energy of rotation of a suspended particle was equal to the mean kinetic energy of translation and both equal to the mean kinetic energy of an isolated moleculewas correct, Einstein succeeded in obtaining an equation which gave the root-mean-squared rotation of a spherical suspended particle during time $t$, as a result of the irregular molecular impacts.

$$
\sqrt{\overline{\Delta_{r}^{2}}}=\sqrt{t} \sqrt{\frac{R T}{N} \frac{1}{4 \pi k P^{3}}}
$$

Perrin's stated aim behind this experimental effort was not the confirmation of the molecular theory of Brownian movement, nor another determination of $N$, but the confirmation of the theoretical assumptions underlying Einstein's rotation equation, especially the confirmation of the validity of the theorem of the equipartition of energy for Brownian particles of larger size (Perrin 1909b, 550).

Observations of the rotational Brownian movement had been observed before Einstein, but a quantitative measurement of this rotation appeared to be extremely difficult. Indeed, Einstein's equation predicted a rotation of $100^{\circ}$ per second for particles of size around $1 \mu$ in diameter (assuming for $N$ the value $70.10^{22}$ ). Perrin, however, realized that an experimental measurement 
was possible for the uniform granules of much larger size that he knew how to prepare. For example, for granules of 10 to $15 \mu$ in diameter, the predicted rotation was no more than a few degrees per minute. Perrin used spherical granules of mastic of $13 \mu$ in diameter-which were approximately 100000 times heavier than the granules used in the vertical distribution experiments-in a solution of urea. He took advantage of the fact that some of the granules often contained small inclusion or defects which could observed with a microscope. Perrin was able to measure the distance of the small inclusions from the centre and also dot their successive positions after specific time intervals. This gave him all the elements needed to measure the rotation of the particle around any axis. Substituting in Einstein's equation the average of 200 angular measurements, Perrin got for $N$ the value $65.10^{22}$, which he judged to be more than satisfactory given the difficulties involved in measuring the rotation of the particles. ${ }^{34}$ The numerical concordance became even more striking if one considered the possible a priori values for the mean rotation of a Brownian particle. Perrin's conclusion was that the measurements of the Brownian rotational movement established the validity of the theorem of the equipartition of energy even for Brownian particles 100000 heavier than those examined in the vertical distribution experiments (Perrin 1909c, 92).

\section{The Reaction of the Scientific Community}

Perrin continued to work towards a further solidification of the atomic-molecular reality even after his experimental verification of Einstein's formulae. His efforts were twofold. On the one hand, with the help of his students, Perrin continued his experimental work on Brownian movement. This work was mostly a refinement of his earlier experiments. It involved the variation of the various parameters: nature, viscosity, and density of the intergranular liquid, 
nature and size of the suspended particles, and so on. The aim of this work was not to offer additional support for the molecular theory of Brownian movement, nor to test the mathematical formulae describing the phenomenon, but to provide more accurate values for the various molecular magnitudes (Perrin 1911a, 1911b, Perrin and Bjerrum 1911). ${ }^{35}$ On the other hand, Perrin began an intensive campaign of popularizing and propagandizing his experimental work on Brownian movement and its connection with the additional evidence that was emerging in favor of the molecular hypothesis. The result was a series of research papers, popular articles, and conference presentations, which was concluded with the publication of the book Les Atomes, in 1913 (1910, 1912a, 1912b, 1913a, 1913b).

The response of the scientific community was extremely favorable. ${ }^{36}$ Perrin's work was able to persuade scientists who had previously been skeptic about or who had even explicitly rejected the atomic-molecular theory. Writing in 1968, historian of science Stephen Brush was perplexed by Perrin's success.

[Perrin] was surprisingly successful. In fact, the willingness of scientists to believe in the "reality" of atoms after 1908, in contrast to previous insistence on their "hypothetical" character, is quite amazing.

The evidence provided by the Brownian movement experiments of PERRIN and others seems rather flimsy, compared to what was already available from other sources. The fact that one could determine Avogadro's number and the charge on the electron by one more method seems hardly sufficient to justify such profound metaphysical conclusions. Several independent methods of determining these parameters had been known since 1870 or before, to say nothing of the many 
successes of the kinetic theory in predicting the properties of gases (Brush 1968, 34).

In Section 4, I showed why Perrin think that the determinations of molecular magnitudes made within the context of the kinetic theory of gases, by themselves, did not provide conclusive proof for molecular reality. The brief summary of the state of the atomism debates in the fin-de siècle France (provided in Section 3) and the fact that Perrin had to defend the usefulness of molecular hypotheses in the beginning of the twentieth century, shows that this sentiment towards the existing theoretical determinations of molecular magnitudes was shared by many of his contemporaries. Moreover, from the reactions of the scientific community we can glean specific reasons for Perrin's success. These reasons largely coincide with the elements that we have already identified as responsible for the epistemic strength of Perrin's argument.

First, almost all commentators praised the methodology and ingenuity of Perrin's experimental work, which allowed the measurement of the magnitudes appearing in the mathematical equations of Brownian movement and the subsequent determination of molecular magnitudes. The rationale explicitly expressed by many scholars was that atoms and molecules were real because they could be counted (Nye 1972; 143-52). Second, Perrin's propagandizing efforts were an important reason for his success. Perrin devoted much time in writing papers and attending conferences, in which he was able not only to popularize the significance of his work on Brownian movement, but also to show its relationship with the evidence that was emerging in favor of atomism. Perrin's synthetic approach came at a time when the scientific community was being inundated with a variety of new evidence concerning the particulate structure of matter. The commentators commended Perrin's clear and lucid style of presentation and his convincing combination of all the available evidence to put forward a foolproof argument for molecular 
reality (Nye 1972, 152-65). Third, and foremost, however, it was Perrin's continuous emphasis on the agreement between the values for $N$ determined by independent procedures that provided the knockdown argument for molecular reality. The remarkable numerical agreement gave rise to an extremely strong no-coincidence argument that was used not only to validate the values determined for $N$, but also to argue about the validity of the main auxiliary assumptions underlying the various determinations. In her authoritative scientific biography of Jean Perrin, historian of science Mary Jo Nye has claimed that the most appealing and interesting feature of Perrin's argument was that it meant different things to different people. The numerical agreement on the values for molecular magnitudes was primarily used to support the validity of the determinations and the reality of atoms and molecules, but it could be also used to support the molecular explanation of Brownian movement, the probabilistic interpretation of Carnot's principle, the theorem of the equipartition of energy, the theory of black-body radiation, and so on (Nye 1972, 143-45). ${ }^{37}$

The no-coincidence argument was difficult to resist even by long-life opponents and skeptics of atomism. Perhaps the most important conversion was that of Wilhelm Ostwald, the main representative of the program of Energetics. Ostwald's explicit recantation of his opposition to atomism occurred in the preface of the fourth edition of his Grundriss der Allgemeinen Chemie, in 1909. Ostwald explained that his past rejection of atomism was grounded on the absence of any direct and conclusive experimental proof. He admitted that the agreement of Perrin's measurements on Brownian movement with the postulates of the kinetic theory and J.J. Thomson's measurements of ions in gases provided such a proof (Ostwald 1912, vi). 
Henri Poincaré was another major figure who, although had doubted the possibility and usefulness of a scientific investigation of unobservable entities, became convinced for the reality of atoms and molecules by Perrin's arguments. Poincaré expressed his conviction after getting a firsthand account of the new developments, first at the First Solvay Conference, in 1911, and then again at the conference of the Société Française de Physique, in 1912. In both conferences, Perrin presented the same lengthy discussion, cataloguing in detail les preuves de la réalité moléculaire that had emerged from the investigations of different phenomena and emphasizing the numerical agreement on the value for $N$ (Perrin 1912a, 1913a).

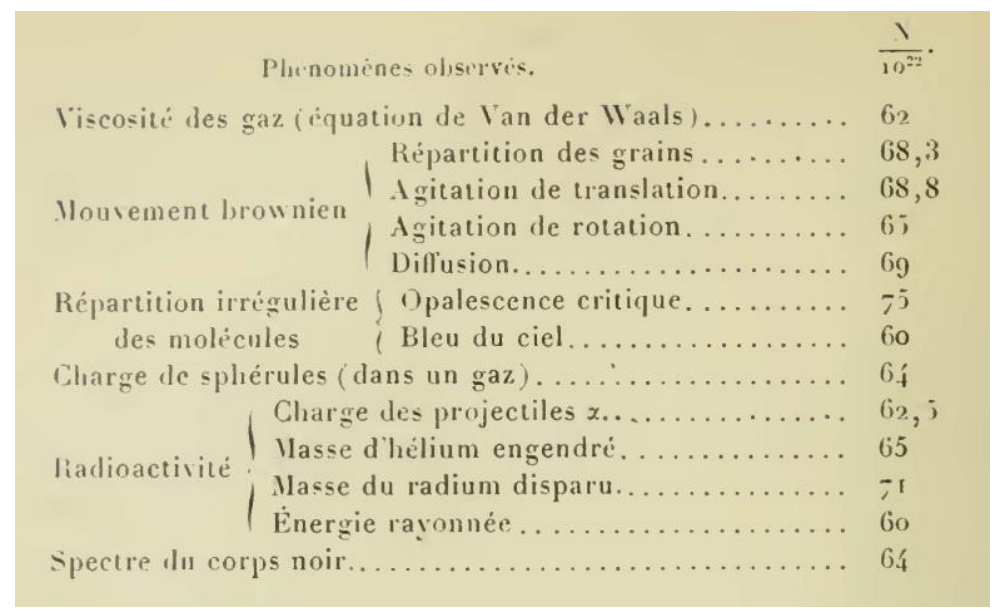

Fig.3

The chart summarizing the numerical agreement on the values for

$N$ determined in the independent investigation of different phenomena, from the conclusion of Perrin's presentation at the conference of the Société Française de Physique, in 1912 (Perrin1913a, 52).

Perrin argued that the invariance of each value for $N$ within the same determination, and the agreement among independent determinations gave to molecular reality the same certainty enjoyed by the principles of Thermodynamics: 
One is seized with admiration in front of the miracle of so precise concordances, starting from phenomena so different. That one finds the same value within each method, when varying as much as possible the parameters of the experiment, and that the numbers thus calculated without ambiguity by these diverse methods coincide, gives to molecular reality as much certainty as the principles of Thermodynamics (Perrin 1912b, 512)

Poincaré's contribution to the 1912 conference was a critical reflection on the papers presented. Not only did Poincaré accept that the evidence presented had conclusively proved the existence of the atoms and the molecules postulated by the kinetic theory, but he also provided an insightful analysis of the epistemic import of this evidence. Poincaré claimed that atoms and molecules had ceased to be convenient fictions; one could even say that they had become observable entities since researchers now knew how to count them. What made it certain that researchers were counting real entities was (a) the close numerical agreement on the values for molecular magnitudes obtained by (b) independent procedures when investigating (c) different phenomena that were manifestations of (d) different properties of the entities under consideration. This numerical agreement gave rise to a remarkable no-coincidence argument for the reality of the entities measured. What made this argument even more forceful was the (e) lack of any discordant results despite the (f) extremely large range of numerical values that were possible in each one of the different investigations (Poincare 1913, 357-59). ${ }^{38}$

Finally, Jacques Loeb, in his influential article "Mechanistic Science and Metaphysical Romance," also emphasized the quantitative nature of the agreement between independent determinations for the epistemic force of the no-coincidence argument. Loeb's article aimed to present his conception of mechanistic science and its difference from non-mechanistic ways of 
thinking. Loeb presented Perrin's argument for molecular reality as the paradigmatic form of the methodology followed in mechanistic science and as the methodological approach that ought to be followed in the biological and life sciences to replace the last vestiges of metaphysical and romantic thinking. Loeb explained that Perrin's argument removed over one hundred years of doubt over the existence of molecules and set physics on a clear mechanistic path. Analyzing the features of Perrin's argument that allowed it to perform such a feat, Loeb cited: (a) the quantitative (numerical) determinations of molecular magnitudes (Loeb 1915, 768), (b) the fact that these determinations were done by different physicists, working in different fields of physics (Loeb 1915, 770), and (c) the very close numerical agreement between the different determinations:

This method [of mechanistic science] is essentially quantitative. The ancients also spoke of discrete ultimate particles, but what they said was not based upon experiment or quantitative measurement. The basis for an atomic theory was laid by Dalton's measurements a century ago, and the theory was proved by the measurements in the last decade previously referred to. Mechanistic science, then, tests the correctness of an attempted visualization by a comparison of the observed and the calculated values. A merely qualitative experimental corroboration of a theory may be, and often is, insufficient. If the agreement between observed and expected facts is not only qualitative but also numerically correct, the probability of an accident becomes comparatively small. The more closely the calculated and observed values agree, the smaller the probability that the theory was wrong. And if entirely different methods lead to the same 
quantitative values, the probability of correctness approaches absolute truth

(Loeb 1915, 781-82, my italics).

In a letter to Perrin, Loeb reiterated his high praise: "I hardly need to tell you that your proof of the real existence of molecules will live in science as an epoch-making discovery - and not only in science but in our general philosophical view of the universe." ${ }^{39}$

\section{Conclusion}

The almost universal and uncritical acceptance of molecular reality after nearly one hundred years of controversy made Perrin's argument the exemplar of scientific argumentation for the next generations of researchers. It is not surprising, therefore, that many philosophers have tried to analyze the reasoning underlying Perrin argument. In doing so, however, most of the philosophers have largely ignored the historical context and temporal development of Perrin's work, and have relied instead on the final versions of Perrin's argument. In addition, rather than aiming at a dispassionate reconstruction of Perrin's argument, the various philosophers have attempted to appropriate Perrin for their own purposes. Therefore, it is also not surprising that they have arrived at different interpretations of his argument for molecular reality.

In this paper, I followed a historicist approach to understand the reasoning underlying Jean Perrin's argument for molecular reality. I showed that what was at stake in early twentieth century atomism debates was not simply the question of whether there was enough evidence to establish the existence of unobservable atoms or molecules, but-even more fundamentally-the questions of whether scientific theories should appeal to such entities and, further, what would count as evidence for their existence. The role of Jean Perrin in the resolution of these questions was crucial. Equipped with a deep knowledge of both the scientific developments of the time and the philosophical issues surrounding the atomism debates, Perrin not only established the atomic- 
molecular hypothesis as a scientific hypothesis amenable to experimental verification, but also provided the much-needed methodological approach for such a verification.

Perrin put forward a no-coincidence argument for the existence of molecules, which was based on the agreement between multiple, independent determinations of Avogadro's number (and, consequently, other molecular magnitudes). The blunt rationale of the argument was the following: it would be a highly improbable coincidence for multiple, independent determinations of molecular magnitudes to achieve concordant results, and yet for there not to be any molecules. The historicist approach followed here, however, shows that there were additional structural elements of Perrin's argument that were responsible for its exceptional strength and, ultimately, for its success. They were the following:

1. The quantitative nature of the various determinations. That is, the various determinations provided specific numerical values for the molecular magnitudes.

2. The close numerical agreement between the various determinations. This agreement became even more striking if one considered the possible values for the molecular magnitudes that could have been the result of each one of the determinations.

3. The number of determinations. There was a (relatively) large number of determinations that converged on the same result.

4. The theoretical independence of the various determinations. The various determinations were based on independent theoretical auxiliary assumptions. In other words, the validity of the theoretical auxiliary assumptions underlying one determination procedure, did not depend on the validity of the assumptions underlying another procedure. 
5. The genetic independence of the various determinations. The various determinations were conducted independently of one another and no effort was made to mutually adjust the numerical values determined by the theory-independent procedures.

6. The variety of the phenomena investigated. The various determinations were based on the investigation of unrelated phenomena, which were observable manifestations of different properties of the entities investigated.

7. The high quality and reliability of some of the determinations.

8. The lack of any discordant results, despite there being a large number of determinations.

9. The ability to conclusively resolve the objections and the discordant results that challenged Perrin's determination of molecular magnitudes.

These structural elements of Perrin's use of the multiple determination strategy gave rise to an exceptionally strong no-coincidence argument that was used to argue for both the validity of the molecular magnitudes determined and the validity of the (theoretical and experimental) auxiliary assumptions underlying the various determination procedures.

Finally, the structural elements of Perrin's no-coincidence argument for molecular reality, in tandem with the study of other cases of multiple determination-from past or current sciencecan be used to develop a conceptual framework for evaluating the epistemic strength of concrete applications of the multiple determination strategy. This framework, more nuanced than the blunt rationale, can help to resolve the existing disagreements regarding the relevance and the epistemic import of multiple determination strategy in scientific research (Coko 2015b; 2019). 


\section{Acknowledgments}

Christopher ChoGlueck and Jutta Schickore provided helpful feedback on earlier versions. I would like to thank three anonymous referees for their insightful comments and suggestions.

\section{References}

Achinstein, Peter. 2001. The Book of Evidence. Oxford: Oxford University Press.

Brooke, John H. 1981. “Avogadro’s Hypothesis and its Fate: A Case-Study in the Failure of Case-Studies.” History of Science 19 (4): 235-73.

Brush, Stephen G. 1968. “A History of Random Processes: I. From Brown to Perrin.” Archive for History of Exact Sciences 5 (1): 1-36.

Cartwright, Nancy. 1983. How the Laws of Physics Lie. Oxford: Oxford University Press.

Cartwright, Nancy. 1991. "Replicability, Reproducibility and Robustness: Comments on Harry Collins." History of Political Economy 23: 143-55.

Chalmers, Alan. 2009. The Scientist's Atom and the Philosopher's Stone: How Science Succeeded and Philosophy Failed to Gain Knowledge of Atoms. Boston Studies in the Philosophy of Science 279. Dordrecht: Springer.

---. 2011. “Drawing Philosophical Lessons from Perrin’s Experiments on Brownian Motion: A Response to van Fraassen.” The British Journal for the Philosophy of Science 62 (4): 711-32. Chaudesaigues. 1908. “Le Mouvement Brownien et le Formule d’Einstein.” Comptes Rendus 147: $1044-46$.

Clark, Peter. 1976. “Atomism Versus Thermodynamics." In Method and Appraisal in the Physical Sciences, ed. Colin Howson, 41-105. Cambridge: Cambridge University Press. 
Coko, Klodian. 2015a. “Epistemology of a Believing Historian: Making Sense of Duhem's Antiatomism." Studies in History and Philosophy of Science A 50: 71-82.

Coko, Klodian. 2015b. The Structure and Epistemic Import of Empirical Multiple Determination in Scientific Practice. Dissertation. Indiana University.

Coko, Klodian. 2019. "Towards a Mutually Beneficial Integration of History and Philosophy of Science: The Case of Jean Perrin.” In The Past, The Present, The Future of iHPS, ed. Emily Herring et al. London: Routledge.

Collins, Harry M. 1985. Changing Order: Replication and Induction in Scientific Practice.

Chicago: The University of Chicago Press.

Cotton, Aimé. 1908. "Recherches Récentes sur les Mouvements Browniens." La Revue du Mois 5: 737-41.

Culp, Sylvia. 1994. "Defending Robustness: The Bacterial Mesosome as a Test Case." PSA: Proceedings of the Biennial Meeting of the Philosophy of Science Association 1: 46-57.

---. 1995. "Objectivity in Experimental Inquiry: Breaking Data-Technique Circles.” Philosophy of Science 62 (3): 438-58.

Dalton, John. 1808. A New System of Chemical Philosophy. Manchester: S. Russell. de Broglie, Louis. 1945. La Réalité des Molécules et L'CEuvre de Jean Perrin. Paris: GauthierVillars.

Duclaux, Joseph. 1904. Recherches sur les Substances Collö̈dales, Paris: Laval.

---. 1908. "Pression osmotique et mouvement brownien." Comptes Rendus 147: 131-34.

Duhem, Pierre. 1893. "Physique et métaphysique." Revue des Questions Scientifiques 33: 99-133

---. 1894. “Quelques Réflexions au Sujet de la Physique Expérimentale.” Revue des Questions Scientifiques 36: 179-229. 
---. 1906. La théorie physique, son objet et sa structure. Paris: Chevalier \& Riviére Einstein. Albert. 1956a. "On the Movement of Small Particles Suspended in a Stationary Liquid Demanded by the Molecular-Kinetic Theory of Heat." In Investigations on the Theory of the Brownian Movement by Albert Einstein, ed. R. Fürth, trans. A. D. Cowper, 1-18. Dover Publications.

---. 1956b. "On the Theory of the Brownian Movement." In Investigations on the Theory of the Brownian Movement by Albert Einstein, ed. R. Fürth, trans. A. D. Cowper, 19-36. Dover Publications.

Fisher, Nicholas. 1982. "Avogadro, the Chemists, and Historians of Chemistry: Part 1." History of Science 20 (2): 77-102.

Fleck, George M. 1963, “Atomism in Late Nineteenth Century Physical Chemistry.” Journal of the History of Ideas, 24: 106-14.

Gardner, Michael. 1979. "Realism and Instrumentalism in 19th-century Atomism." Philosophy of Science 46: 1-34.

Glymour, Clark. 1975. "Relevant Evidence.” The Journal of Philosophy 72 (14): 403-26.

---. 1980. Theory and Evidence. Princeton: Princeton University Press.

Gouy, Léon. 1888. “Note sur le Mouvement Brownien.” Journal de Physique 7: 561-564.

---. 1889. "Sur le Mouvement Brownien." Comptes Rendus 109: 102-105.

---. 1895. "Le Mouvement Brownien et les Mouvements Moléculaires." Revue Générale des

Sciences 6: 1-7.

Hacking, Ian. 1983. Representing and Intervening: Introductory Topics in the Philosophy of Natural Science. Cambridge: Cambridge University Press. 
Harman, Gilbert H. 1965. “The Inference to the Best Explanation.” The Philosophical Review 74 (1): 88-95.

Henri, Victor. 1908. “Étude Cinématographique des Mouvement Brownien.” Comptes Rendus, 146: 1024-1026.

Hudson, Robert G. 2014. Seeing Things: The Philosophy of Reliable Observation. Oxford: Oxford University Press.

Kerker, Milton. 1976. “The Svedberg and Molecular Reality.” Isis 67 (2): 190-206.

Klein, Ursula. 2003. Experiments, Models, Paper Tools: Cultures of Organic Chemistry in the Nineteenth Century. Stanford: Stanford University Press.

Kosso, Peter. 1988. "Dimensions of Observability." The British Journal for the Philosophy of Science 39 (4): 449-67.

Langevin, Paul. 1908. "Sur la Théorie du Mouvement Brownien." Comptes Rendus 146: 530-33. Loeb, Jacques. 1915. "Mechanistic Science and Metaphysical Romance." Yale Review 4: 76685.

Maiocchi, Roberto. 1990. “The Case of Brownian Motion.” The British Journal for the History of Science 23 (3): 257-83.

Mayo, Deborah G. 1986. "Cartwright, Causality, and Coincidence." PSA: Proceedings of the Biennial Meeting of the Philosophy of Science Association 1986 (1): $42-58$.

--- 1996. Error and the Growth of Experimental Knowledge. Chicago: University of Chicago Press.

Mazliak, Laurent. 2007. “Borel, probability and La Revue du Mois.” Electronic Journ@l for History of Probability and Statistics 3 (1): June 2007. 
McMullin, Ernan. 1970. “The History and Philosophy of Science: A Taxonomy.” In Minnesota

Studies in the Philosophy of Science, ed. Roger H. Stuewer, 12-67. Minneapolis: University of Minnesota Press.

Needham, Paul. 2004. "Has Daltonian Atomism Provided Chemistry with Any Explanations?” Philosophy of Science 71: 1038-47.

Nye, Mary J. 1972. Molecular Reality: A Perspective on the Scientific Work of Jean Perrin. New York: American Elsevier Company.

Nye, Mary Jo ed. 1984. The Question of the Atom: From the Karlsruhe Congress to the First Solvay Conference. 1860-1911. A Selection of Primary Sources. Los Angeles and San Francisco: Tomash Publishers.

Ostwald, Wilhelm. 1912. Outlines of General Chemistry. Trans. W. W. Taylor. London: MacMillan.

Paul, Harry W. 1972. "The Crucifix and the Crucible: Catholic Scientists in the Third Republic." The Catholic Historical Review 58: 195-219.

Perrin, Jean. 1895. "Nouvelles propriétés des rayons cathodiques.” Comptes Rendu 121: 1130-34.

---. 1897. Rayons Cathodiques et Rayons de Röntgen: Étude Expérimentale. Paris: GauthierVillars et fils.

---. 1901. “Les Hypothèses Moléculaires.” Revue Scientifique 15 (15): 449-61.

---. 1903. Traité de Chimie Physique: Les Principes. Paris: Gauthier-Villars.

---. 1905. “Mécanisme de L'électrisation de Contact et Solutions Colloïdales.” Journal de Chimie Physique 3: 50-110.

---. 1906a. "Le Contenu Essentiel des Principes de la Thermodynamique." Bullétin de la Société de Philosophie 6: 81-111.

---. 1906b. “La Discontinuité de la Matière.” Revue de Mois 1: 323-43. 
---. 1908a. "L'agitation Moléculaire et le Mouvement Brownien." Comptes Rendus 147: 967-70.

---. 1908b. "La Loi de Stokes et Le Mouvement Brownien.” Comptes Rendus 147: 475-76.

---. 1908c. “L'origine du Mouvement Brownien." Comptes Rendus 147: 530-32.

---. 1908d. “Grandeur des Molécules et Charge de L'électron.” Comptes Rendus 147: 594-96.

---. 1908e. "Peut-on Peser un Atome avec Précision?" La Revue du Mois 6: 513-38.

---. 1909a. "Mouvement Brownien et Molécules." Journal de Physique Théorique et Appliquée

9: 5-39.

---. 1909b. “Le Mouvement Brownien de Rotation.” Comptes Rendus 149: 549-551.

---. 1909c. "Mouvement Brownien et Réalité Moléculaire." Annales de Chimie et de Physique

18: $1-114$.

---. 1910. “Mouvement Brownien et Molécules.” Journal de Physique Théorique et Appliquée 9: 5-39.

---. 1911a. "Les Déterminations des Grandeurs Moléculaires." Comptes Rendus 152: 1165-68.

---. 1911b. "Les Grandeurs Moléculaires (nouvelles mesures).” Comptes Rendus 152: 1380-82.

---. 1912a. “Les Preuves de la Réalité Moléculaire.” In La Théorie du Rayonnement et les

Quanta: Rapports et discussions de la Réunion tenue à Bruxelles, du 30 Octobre au 3 Novembre 1911, ed. Paul Langevin and Maurice de Broglie, 153-251. Paris: Gauthiers-Villars.

---. 1912b. "L'agitation Moléculaire." La Revue du Mois 14: 513-40.

---. 1913a. “Les Preuves de la Réalité Moléculaire.” In Les Idées Modernes sur la Constitution de la Matière, 1-53. Paris: Gauthier-Villars.

---. 1913b. Les Atomes. Paris: Libraire Félix Alcan.

---. 1923. Notice sur les Travaux Scientifiques de M. Jean Perrin, Toulouse: Édouard Privat. 
---. 1926. "Discontinuous Structure of Matter.” Nobel Prize Lecture

https://www.nobelprize.org/prizes/physics/1926/perrin/lecture/

Perrin, Jean and Niels Bjerrum. 1911. "L’agitation Moléculaire dans les Fluids Visqueux."

Comptes Rendus 152: 1569-171.

Perrin, Jean and Dabrowski. 1909. "Mouvement Brownien et Constants Moléculaires." Comptes

Rendus 149: 477-79.

Poincaré, Henri. 1913. “Les Rapports de la Matière et de L’Éther.” In Les Idées Modernes sur la Constitution de la Matière, 357-70. Paris: Gauthier-Villars.

Psillos, Stathis. 2011b. "Making Contact with Molecules: On Achinstein and Perrin.” In

Philosophy of Science Matters: The Philosophy of Peter Achinstein, ed. Gregory J. Morgan, 177-

90. Oxford: Oxford University Press.

---. 2011b. "Moving Molecules above the Scientific Horizon." Journal for General Philosophy of Science 49: 339-63.

---. 2014. "The View from Within and the View from Above: Looking at van Fraassen's Perrin." In Bas van Fraassen's Approach to Representation and Models in Science, ed. Wenceslao J. Gonzalez, 143-66, Dordrecht: Springer.

Raman, Varadaraja V. 1970. "Jean Baptiste Perrin: Advocate for the Atoms." The Physics Teacher 8: 380-86.

Richards, Robert J. 1992. "Arguments in a Sartorial Mode, or the Asymmetries of History and Philosophy of Science." PSA: Proceedings of the Biennial Meeting of the Philosophy of Science Association 1992 (2): 482-489.

Rocke, Alan. 1984. Atomism in the Nineteenth Century: From Dalton to Cannizzaro. Ohio: Ohio State University Press. 
Salmon, Wesley. 1978. "Why ask "Why?”? An Inquiry concerning Scientific Explanation." Proceedings and Addresses of the American Philosophical Association 51 (6): 683-705.

---. 1984. Scientific Explanation and the Causal Structure of the World. Princeton: Princeton University Press.

Schilpp, Paul A., ed. 1949. Albert Einstein: Philosopher-Scientist. The Library of Living

Philosophers 7. Evanston, IL: The Library of Living Philosophers.

Soler, Léna 2012. "Introduction: The Solidity of Scientific Achievements: Structure of the Problem, Difficulties, Philosophical Implications." In Characterizing the Robustness of Science: After the Practice Turn in Philosophy of Science, Boston Studies in the Philosophy of Science 292, ed. Léna Soler et al., 1-60. Dordrecht: Springer.

Soler, Léna, Emiliano Trizio, Thomas Nickles, William C. Wimsatt eds. 2012. Characterizing the Robustness of Science: After the Practice Turn in Philosophy of Science, Boston Studies in the Philosophy of Science 292. Dordrecht: Springer.

Stegenga, Jacob. 2009. "Robustness, Discordance, and Relevance." Philosophy of Science 76 (5): 650-61.

van Fraassen, Bas C. 2009. "The Perils of Perrin, in the Hands of Philosophers." Philosophical Studies 143: 5-24.

Wimsatt, William, C. 1981. “Robustness, Reliability, and Overdetermination.” In Scientific Inquiry and the Social Sciences, ed. Marilynn B. Brewer and Barry E. Collins, 124-163. San Francisco: Jossey-Bass.

Woodward, Jim. 2006. "Some Varieties of Robustness.” Journal of Economic Methodology 13 (2): $219-40$. 
${ }^{1}$ Perrin presented similar summarizing charts in the concluding sections of all his subsequent presentations of the argument for molecular reality (Perrin 1910, 38; 1912, 249; 1913a, 52; 1913b, 289).

2 "I believe it impossible that a mind clear of all preconception can reflect on the extreme diversity of the phenomena which thus converge towards the same result, without experiencing a very strong impression, and I think that from now on it will be difficult to defend by rational arguments a hostile attitude towards molecular hypotheses" (Perrin 1909c, 111). Unless otherwise indicated, translations are mine.

${ }^{3}$ The terms used to refer to this epistemic strategy differ from author to author. Some of the most frequently used terms are: 'robustness,' 'experimental robustness,' 'measurement robustness,' 'triangulation,' and 'independent confirmation.' I use the term 'multiple determination,' because: (a) it is closer to Perrin's own terminology, (b) it is more transparent and less technical than the other terms, and (c) it helps avoiding certain confusions. For example, the term 'robust,' whenever is used with some sort of rigor-and not simply as synonymous to 'solid' or 'strong'refers to something that remains invariant to perturbations. Some authors justify the use of the term 'robustness' in Perrin's case, because they consider the value for $N$ to have remained invariant across the various determination procedures. As it will be clear in the rest of this paper, however, the latter is not the case. In addition, the use of the term 'robustness' blurs an epistemically important distinction between two different epistemic strategies used by Perrin: Namely, the invariance of the value for $N$ within the same determination procedure to the variation of the different parameters (which is indeed a form of robustness), and the agreement on the values for $N$ obtained by genetically-independent and theory-independent procedures. Perrin used the first to establish the existence (or absence) of causal dependencies, and the latter 
to construct a no-coincidence argument. Elsewhere (Coko 2015b), I provide additional reasons for distinguishing robustness (understood as invariance to perturbations) from the multiple determination strategy. I will use the term 'triangulation' to refer to the determination of the same result by two independent procedures. Triangulation is the simplest form of multiple determination. Finally, we should keep in mind that the term 'multiple determination' is used here to refer to a particular epistemic strategy. I maintain that the analysis of Perrin's use of this strategy, provided here, does not depend on the term used to refer to it.

${ }^{4}$ van Fraassen's interpretation has been criticized in detail by Alan Chalmers (2011) and Psillos (2014). In addition, there are passages in Perrin's work (1908e, 538; Perrin 1909c, 111-13) which flatly contradict van Fraassen's interpretation.

${ }^{5}$ Psillos is a notable exception.

${ }^{6}$ This is not to say that I do not share specific views at the meta-level, regarding the proper relationship between philosophy of science and (historical) accounts of scientific practice. Elsewhere (Coko 2019), I describe these views in detail.

${ }^{7}$ Rocke (1984) offers a detailed account of nineteenth century chemical atomism. Chalmers (2009, Chapters 9-11) offers a comprehensive and accessible account of nineteenth century atomic theories. Nye (1984) provides a selection of the most important primary sources on nineteenth century atomism.

${ }^{8}$ Brooke (1981) and Fisher (1982), however, show that the actual historical situation was far more complex.

${ }^{9}$ Clark (1976) and Fleck (1965) provide an overview of the problems facing the kinetic program during the second half of the nineteenth century. 
${ }^{10}$ Nye $(1972,29-38)$ and Paul (1972) describe in detail the debates over the "bankruptcy of science".

${ }^{11}$ In her classic scientific biography of the French physicist, Nye (1972, 52-64) situates Perrin in the scientific and intellectual milieu of the time and shows how this environment of turmoil affected his professional goals.

${ }^{12}$ de Broglie (1945), Raman (1968), and Nye (1972) provide additional biographical facts. ${ }^{13}$ Perrin's exceptional experimental work on cathode and X-rays earned him, in 1898, the assignment of organizing a new course de chimie physique at the Sorbonne. It was a position that Perrin kept until 1939. He first taught the class as chargé de course and later, beginning in 1910, as full professor. Perrin devoted much of the time following his appointment, on establishing a new laboratory and writing a new textbook on physical chemistry. Les Principes was intended as the first volume of a series that would present and discuss the most important topics of study of the new field.

${ }^{14}$ Perrin uses the singular 'molecular hypothesis' in the widest of senses to refer to the hypothesis that matter has a particulate structure and it is not continuous and infinitely divisible all the way down. He recognized, however, that whereas one could imagine only one continuous structure for matter, a discontinuous structure could be realized in many different ways (1901, 41). Perrin uses the plural 'molecular hypotheses' to refer mostly to the claims about the nature of the particulate structure of matter made in the context of nineteenth century chemical atomism and the kinetic theory of gases. I use the term 'atomic-molecular hypotheses' to refer to the same hypotheses.

${ }^{15}$ Perrin (1909c, 22-25; 1913b, 46-49), provide the details of the determinations of Avogadro number made in the context of the kinetic theory of gases. 
${ }^{16}$ This was a strategy that Perrin had already implemented, although with limited success, in his dissertation (Perrin 1897, 17-19, 47)

${ }^{17}$ Perrin's argument appeared in the first issue of the first volume of the journal La Revue $d u$ Mois, a scientific and literary journal founded by the French mathematician Emilé Borel-who made important, although not well-known, contributions to the kinetic theory of gases-and his wife Marguerite Appell (known as Camille Marbo), after Borel received 10000 francs from the Academy of Sciences of Paris (Mazliak 2007, 1). A programmatic text probably written by Borel and Marbo described the nature and scope of the journal: "The number and the importance of questions that may be dealt with by the scientific method is increasing every day. It seemed to us that it was possible to conceive a publication based on this method, though not especially technical, and having as its essential aim to contribute to the development of general ideas by the exposition and the discussion of the progresses obtained in the knowledge of facts and of the movements of ideas which are their consequence. La Revue du Mois tries to be this publication. Its main aim is to be a journal of free discussion, permitting the expression of any opinion with a scientific basis with complete independence. (...) The contents of La Revue du Mois are therefore sufficiently varied for any instructed person to be interested in it" (Mazliak 2007, 1). I would like to thank one of the anonymous reviewers, who pointed out this historical context to me.

${ }^{18}$ The qualitative triangulation also failed to establish kinetic-molecular motion as the unique cause of Brownian movement.

${ }^{19}$ Van't Hoff had shown that all dissolved substances exert on a semi-permeable membrane, which stops them but allows the solvent to pass, an osmotic pressure equal to the pressure that would be developed in the same volume by a gaseous substance containing the same number of 
gram-moles. Assuming the validity of Avogadro's hypothesis, this meant that the same number of any molecules-either as a gas or in a solution-when enclosed in the same volume and at the same temperature exert the same pressure on the walls confining them.

${ }^{20}$ Perrin's approach was a direct application of his early methodological views. For Perrin, there was no reason to impose a rigid distinction between what is directly observable and what is not. The limit between the two is contingent on human perceptual abilities and on the current stage of technological development. It was, therefore, legitimate to examine whether the conclusions about the unobservable atoms and molecules, which were derived in a deductive manner in the context of the kinetic theory of gases, continued to hold at an order of magnitude amenable to empirical investigation.

${ }^{21}$ The previous sentence reads: "This brilliant hypothesis [the kinetic-molecular motion as a cause of Brownian movement], however, was not established and it was not certain that it accounted, even as an order of magnitude, for the phenomenon in question.” This confirms the interpretation given in the previous section. Namely, that although Gouy's experiments had excluded all the influences external to the liquid from being a cause of Brownian movement, and although one could arrive at a molecular explanation of the phenomenon from a purely qualitative consideration of its observable features, only a quantitative triangulation could establish the kinetic-molecular motion as being the (unique) cause of Brownian movement. At the time when Perrin was conducting his first experiments, most colloid chemists, although they accepted the relevance of kinetic-molecular motion for Brownian movement, were not convinced that it provided a total explanation of the phenomenon; they preferred a combination of kineticmolecular motion and electrostatic interactions between the particles (Nye 1972, 102). 
${ }^{22}$ Perrin assumed that the ratio $\frac{n_{0}}{n}$ of the concentration of granules at two different levels was equal to the mean ratio of the number of granules visible by microscope at these two levels. ${ }^{23}$ Duclaux maintained that kinetic-molecular motion in combination with electrostatic interactions between the particles provided a better explanation of Brownian movement (Duclaux 1908, 133).

24 "In short, the three procedures employed justify themselves by their concordance" (Perrin 1909c, 51).

25 "I have already, here [in the same journal], indicated in a qualitative fashion, the conceptions that serve as its [the Atomistics' program] point of departure. I shall therefore resume them briefly, aiming this time to arrive at precise conceptions, expressed by numbers" (Perrin 1908e, 513 , my italics).

${ }^{26}$ Chalmers $(2011,720-23)$ provides a detailed account of the independence of the assumptions involved in calculating the magnitudes appearing in Perrin's vertical distribution equation from the theoretical assumptions of the kinetic theory of gases.

${ }^{27}$ See, for example, the conclusion of Perrin's 1909c monograph.

${ }^{28}$ Perrin's reasoning is not necessarily troublesome or vicious. Since the emergence of the problem of the experimenters' regress-which can be considered as a more general version of the Duhem thesis-philosophers have been aware of the interdependency existing between the correctness of an experimental result and the reliability of the experimental procedure used to establish that result: a correct experimental result is generally considered to be one produced with a reliable experimental procedure, but a reliable experimental procedure is the one that produces the correct result (Collins 1985). In other words, we do not know if we have obtained the correct result unless we have used a reliable procedure, but we do not know if we have used a 
reliable procedure unless we have obtained the correct result. Insofar as the no-coincidence argument from multiple determination helps to break the regress by arguing about the validity of the result, it can also be used to argue about the reliability of the procedures (Coko 2015b, Chapter 5).

29 "It seems to me that to this reader, who I have perhaps encountered in myself, I could reply like this: «Every time that two phenomena give concordant numbers for a molecular magnitude, one obtains the expression of a reality by eliminating the molecular magnitude variable between the two relations that gave it. For example, in obtaining concordant values from the observation of Brownian movement and from that of black body radiation, at the same time we obtain an unexpected relation between the black body radiation and the Brownian movement, which we will express whenever we will want in a quantitative form. And this is perceptible reality. But we should be careful not to limit ourselves to this statement which would remain barren and cold if we no longer kept track of the discovery process. It would be worth as much as cutting off one's arm once one has picked a fruit»." (See also, Perrin 1909c, 91-92).

30 "[I]t is a simple question of patience and attention" (Perrin 1908e, 532).

${ }^{31}$ See Soler (2012) for a discussion.

${ }^{32}$ By the time his work was to appear in press, however, Einstein became aware of the importance of the phenomenon of 'Brownian molecular motion' for his theoretical work (Einstein 1956, 1).

${ }^{33}$ Kerker (1976) provides an account of Svedberg experimental work on Brownian movement. Perrin, Einstein, and Langevin criticized both the theoretical and the experimental aspects of Svedberg's work (Nye 1972, 127; Einstein 1956, 63; Perrin 1909c, 74; Langevin 1908, 533) 
${ }^{34}$ As Perrin indicated, Einstein's formula predicted for the mean-root-square rotation, assuming $N$ equal to $70.10^{22}$, the value $14^{0}$, whereas the experimentally found value was $14,5^{0}$.

${ }^{35}$ Perrin followed this line of research until 1913, when he found a new and more promising way to investigate the fine structure of matter in the experimental study of thin films (Perrin 1923). ${ }^{36}$ Nye (1972, Chapter 4) provides a detailed account.

${ }^{37}$ See also the conclusions derived by Perrin himself (1909c, 113-14)

${ }^{38}$ Poincare was careful to distinguish the epistemic strength of this new, quantitative form of nocoincidence argument from the epistemic force of traditional "no-coincidence" arguments. Especially from the "no-coincidence" argument emerging when a theoretical hypothesis originally invoked to explain a certain kind of (qualitative) facts was, unexpectedly, found able to explain a different class of (qualitative) facts. According to Poincare, in most cases, a hypothesis cannot explain different kinds of facts from the ones it was originally invoked to explain without the aid of more or less $a d$ hoc assumptions that are added to make the hypothesis compatible with the new facts. Therefore, one cannot infer the truth of a theoretical hypothesis from its ability to explain "different" kinds of facts (Poincaré 1913, 357-58)

${ }^{39}$ Letter from Loeb to Perrin, dated August 7, 1918. As quoted in Nye 1972, 161. 